K. Yoshioka

Nagoya Math. J.

Vol. 154 (1999), 73-102

\title{
SOME NOTES ON THE MODULI OF STABLE SHEAVES ON ELLIPTIC SURFACES
}

\author{
KŌTA YOSHIOKA
}

\begin{abstract}
In this paper, we shall consider the birational structure of moduli of stable sheaves on elliptic surfaces, which is a generalization of Friedman's results to higher rank cases. As applications, we show that some moduli spaces of stable sheaves on $\mathbb{P}^{2}$ are rational. We also compute the Picard groups of those on Abelian surfaces.
\end{abstract}

\section{$\S 0$. Introduction}

Let $X$ be a smooth projective surface over $\mathbb{C}$ and $H$ an ample divisor on $X$. Let $M_{H}\left(r, c_{1}, \Delta\right)$ be the moduli of stable sheaves $E$ of rank $r$ on $X$ with $c_{1}(E)=c_{1} \in \operatorname{NS}(X)$ and $\Delta(E)=\Delta$, where $\Delta(E):=$ $c_{2}(E)-\{(\operatorname{rk}(E)-1) / 2 \operatorname{rk}(E)\}\left(c_{1}(E)^{2}\right)$. In this note, we shall consider the moduli spaces on elliptic surfaces. Let $\pi: X \rightarrow B$ be an elliptic surface such that every singular fibre is irreducible. We denote the algebraic equivalence class of fibres by $f$. We assume that $X$ is regular, the intersection number $\left(c_{1}, f\right)$ is odd and $H$ is sufficiently close to $f$. Then Friedman [F] showed that $M_{H}\left(2, c_{1}, \Delta\right)$ is birationally equivalent to $S^{n}\left(J^{d} X\right)$, where $n=\operatorname{dim} M_{H}\left(2, c_{1}, \Delta\right) / 2,2 d+1=\left(c_{1}, f\right)$ and $J^{d} X$ is an elliptic surface over $B$ whose generic fibre is the set of line bundles of degree $d$. In this note, we shall generalize it to the case where $r$ and $\left(c_{1}, f\right)$ are relatively prime.

As an application, we shall show that $M_{H}(r, k H, \Delta)$ is a rational variety if $(X, H)=\left(\mathbb{P}^{2}, \mathcal{O}_{\mathbb{P}^{2}}(1)\right)$ and $(r, 3 k)=1$. We also consider moduli spaces on Abelian surfaces. In particular, we shall find a set of generators of $H^{2}\left(M_{H}\left(r, c_{1}, \Delta\right), \mathbb{Z}\right)$. For general surfaces, Li [Li1], [Li2] considered the structure of $H^{i}\left(M_{H}\left(2, c_{1}, \Delta\right), \mathbb{Q}\right), i \leq 2$ and $\operatorname{Pic}\left(M_{H}\left(2, c_{1}, \Delta\right)\right) \otimes \mathbb{Q}$ for $\Delta \gg 0$. For the integral cohomologies, Mukai [Mu3], [Mu5] and O'Grady $[\mathrm{O}]$ investigated the structure of $H^{2}\left(M_{H}\left(r, c_{1}, \Delta\right), \mathbb{Z}\right)$ and the Picard group, if $X$ is a K3 surface. By the same method as in [Y2], we get a set of generators of $H^{2}\left(M_{H}\left(r, c_{1}, \Delta\right), \mathbb{Z}\right)$, if $X$ is a ruled surface. Our results for Abelian

Received July 8, 1996. 
surfaces are similar to these results.

In section 1 , we shall consider the birational structure of $M_{H}\left(r, c_{1}, \Delta\right)$. Our method is the same as that in Friedman [F] and Maruyama [Ma2]. That is, we shall use elementary transformations. For simplicity, we assume that $X$ is regular. Let $E$ be a member of $M_{H}\left(r, c_{1}, \Delta\right)$. Since $H$ is sufficiently close to the fibre, $\left.E\right|_{\pi^{-1}(\eta)}$ is a stable vector bundle on the generic fibre $\pi^{-1}(\eta)$. Then there is a stable vector bundle $E_{1}$ on $X$ such that $\left.E_{1}\right|_{l}$ is semi-stable in the sense of Simpson [S] for all fibres $l$, and $E$ is obtained from $E_{1}$ by successive elementary transformations along coherent sheaves of pure dimension 1 on fibres. Let $E_{2}$ be a stable vector bundle such that $\left.\left.E_{2}\right|_{\pi^{-1}(\eta)} \cong E_{1}\right|_{\pi^{-1}(\eta)},\left.E_{2}\right|_{l}$ is semi-stable in the sense of Simpson and $\left.\left.\operatorname{det} E_{2}\right|_{l} \cong \operatorname{det} E_{1}\right|_{l}$ for all fibres $l$. By using the irreducibility of $l$, we shall show that $E_{2} \cong E_{1} \otimes \pi^{*} L$, where $L \in \operatorname{Pic}(B)$. Then we can easily show that $S^{n}\left(J^{d} X\right)$ is birationally equivalent to an irreducible component of $M_{H}\left(r, c_{1}, \Delta\right)$, where $n=\operatorname{dim} M_{H}\left(r, c_{1}, \Delta\right) / 2$ and $d$ is an integer. By the dimension counting of non-locally free part (cf. [Y1, Thm. 0.4]), we see that every irreducible component contains vector bundles (In fact, the non-locally free part is of codimension $r-1)$. Let $E$ be a vector bundle of $M_{H}\left(r, c_{1}, \Delta\right)$. We note that $\operatorname{Ext}^{2}(E, E(-l))_{0} \cong \operatorname{Hom}\left(E, E\left(K_{X}+l\right)\right)_{0}^{\vee}=0$ for all fibres $l$, where $\operatorname{Ext}^{i}(E, E(D))_{0}$ is the trace free part of $\operatorname{Ext}^{i}(E, E(D))$. Then $\operatorname{Ext}^{1}(E, E)_{0} \rightarrow \operatorname{Ext}^{1}\left(\left.E\right|_{l},\left.E\right|_{l}\right)_{0}$ is surjective. Considering the deformation space of $\left.E\right|_{l}$, we shall show that $M_{H}\left(r, c_{1}, \Delta\right)$ is birationally equivalent to $S^{n}\left(J^{d} X\right)$.

In section 2 , we shall treat the moduli spaces on $\mathbb{P}^{2}$. Let $V \subset H^{0}\left(\mathbb{P}^{2}, K_{\mathbb{P}^{2}}^{\vee}\right)$ be a linear pencil which contains an elliptic curve $C$. Since $\left(K_{\mathbb{P}^{2}}, H\right)<0$, we can deform $E \in M_{H}\left(r, c_{1}, \Delta\right)$ to a sheaf $E^{\prime} \in M_{H}\left(r, c_{1}, \Delta\right)$ such that $\left.E^{\prime}\right|_{C}$ is semi-stable. If $\left(c_{1}, H\right)$ and $r$ are relatively prime, then $\left.E^{\prime}\right|_{C}$ is a stable vector bundle. Let $\mathbb{P}^{2} \rightarrow \mathbb{P}^{1}$ be the rational map defined by $V$ and $Y \rightarrow \mathbb{P}^{2}$ the blow-ups of $\mathbb{P}^{2}$ which defines the morphism $Y \rightarrow \mathbb{P}^{1}$. Then $M_{H}\left(r, c_{1}, \Delta\right)$ is birationally equivalent to a component of a moduli space $M_{H^{\prime}}\left(r, c_{1}, \Delta\right)$, where $H^{\prime}$ is an ample divisor on $Y$ which is sufficiently close to the fibre in $\operatorname{NS}(Y)$. Since $M_{H^{\prime}}\left(r, c_{1}, \Delta\right)$ is birationally equivalent to a symmetric product of $Y$, we get that $M_{H}\left(r, c_{1}, \Delta\right)$ is rational. We also prove that the moduli of simple torsion free sheaves on Del Pezzo surfaces are irreducible.

In section 3, we shall consider the moduli spaces on an Abelian surface. We assume that $c_{1} \bmod \operatorname{NS}(X)$ is a primitive element of $\operatorname{NS}(X) / r \operatorname{NS}(X)$. Mukai [Mu1] gave a complete description of $M_{H}\left(r, c_{1}, \Delta\right)$ in the case where 
$\operatorname{dim} M_{H}\left(r, c_{1}, \Delta\right)=2$. Hence we assume that $\operatorname{dim} M_{H}\left(r, c_{1}, \Delta\right) \geq 4$. By using a quasi-universal family [Mu3], we shall construct a set of generators of $H^{i}\left(M_{H}\left(r, c_{1}, \Delta\right), \mathbb{Z}\right)$ for $i=1,2$, where $H$ is a general polarization (Theorem 3.1). Our method is the same as in Göttsche and Huybrechts [G-H], that is, we shall deform $X$ to a product of elliptic curves. Then $M_{H}\left(r, c_{1}, 0\right)$ is isomorphic to $X$ and $M_{H}\left(r, c_{1}, \Delta\right)$ is birationally equivalent to $X \times \operatorname{Hilb}_{X}^{r \Delta}$. Since both spaces have trivial canonical bundles, there are closed subsets $Z_{1} \subset M_{H}\left(r, c_{1}, \Delta\right)$ and $Z_{2} \subset X \times \operatorname{Hilb}_{X}^{r \Delta} \operatorname{such}$ that $\operatorname{codim}\left(Z_{1}\right) \geq 2$, $\operatorname{codim}\left(Z_{2}\right) \geq 2$ and $M_{H}\left(r, c_{1}, \Delta\right) \backslash Z_{1} \cong\left(X \times \operatorname{Hilb}_{X}^{r \Delta}\right) \backslash Z_{2}$. Hence we get an isomorphism $H^{i}\left(M_{H}\left(r, c_{1}, \Delta\right), \mathbb{Z}\right) \cong H^{i}\left(X \times \operatorname{Hilb}_{X}^{r \Delta}, \mathbb{Z}\right), i=1,2$. Constructing a family of stable sheaves parametrized by $X \times \operatorname{Hilb}_{X}^{r \Delta} \backslash Z_{2}$ directly, we shall construct a set of generators of $H^{i}\left(M_{H}\left(r, c_{1}, \Delta\right), \mathbb{Z}\right), i=1,2$. By using deformation of $X$ and the result in [Y4], we shall also show that the Betti numbers of $M_{H}\left(2, c_{1}, \Delta\right)$ are the same as those of $M_{H}(1,0,2 \Delta)$ (Theorem 3.5). We next show that the morphism $M_{H}\left(r, c_{1}, \Delta\right) \rightarrow \operatorname{Pic}^{0}(X) \times X$ defined in [Y2, Sect. 5] is an Albanese map, if $\operatorname{dim} M_{H}\left(r, c_{1}, \Delta\right) \geq 4$. Combining all together, we also describe the Picard group of $M_{H}\left(r, c_{1}, \Delta\right)$ (Theorem 3.6).

I would like to thank Professors A. Ishii and M. Maruyama for valuable discussions.

Notation. Let $X$ be a smooth projective surface over $\mathbb{C}$ and $H$ an ample divisor on $X$. For a scheme $S$, we denote the projection $S \times X \rightarrow S$ by $p_{S}$. We denote the Néron-Severi group of $X$ by $\mathrm{NS}(X)$. For an $x \in$ $\mathrm{NS}(X) \otimes \mathbb{Q}$, we set $P(x):=\left(x, x-K_{X}\right) / 2+\chi\left(\mathcal{O}_{X}\right)$.

For a torsion free sheaf $E$ on $X$, we set

$$
\Delta(E):=c_{2}(E)-\frac{\operatorname{rk}(E)-1}{2 \operatorname{rk}(E)}\left(c_{1}(E)^{2}\right) .
$$

We denote the traceless part of $\operatorname{Ext}^{i}(E, E(D))$ by $\operatorname{Ext}^{i}(E, E(D))_{0}$.

In this note, we use the notion of (semi-)stability of Mumford. Let $M_{H}\left(r, c_{1}, \Delta\right)$ be the moduli of stable sheaves $E$ of rank $r$ on $X$ with $c_{1}(E)=$ $c_{1} \in \mathrm{NS}(X)$ and $\Delta(E)=\Delta$. We denote the open subscheme of $M_{H}\left(r, c_{1}, \Delta\right)$ consisting of stable vector bundles by $M_{H}\left(r, c_{1}, \Delta\right)_{0}$.

\section{$\S 1$. Moduli spaces on elliptic surfaces}

\subsection{Preliminaries}

Let $\pi: X \rightarrow B$ be an elliptic surface. We assume that every fibre is irreducible throughout this setion. We denote a fibre by $f$. Let $\eta$ be the 
generic point of the base curve $B$. Let $J^{d} X \rightarrow B$ be the elliptic surface over $B$ whose generic fibre is the set of line bundles of degree $d$ on $\left.X\right|_{\pi^{-1}(\eta)}$. For a coherent sheaf $E$ on a fibre $l$, we set

$$
\begin{aligned}
\operatorname{rk}(E) & :=\operatorname{length}_{\mathcal{O}_{\eta_{l}}}\left(E \otimes \mathcal{O}_{\eta_{l}}\right), \\
\operatorname{deg}(E) & :=\chi(E),
\end{aligned}
$$

where $\eta_{l}$ is the generic point of $l$.

A coherent sheaf $E$ of pure dimension 1 on a fibre $l$ is semi-stable if

$$
\frac{\chi(F)}{\operatorname{rk}(F)} \leq \frac{\chi(E)}{\operatorname{rk}(E)}
$$

for all subsheaves $F \neq 0$ of $E$.

Lemma 1.1. Let $L$ be a relatively ample divisor on $X$. Let $D$ be a divisor on $X$ such that $(D, f) \neq 0$ and $(D, L+k f)=0$ for some positive number $k$. Then,

$$
\left(D^{2}\right) \leq \frac{-1}{(L, f)^{2}}\left(\left(L^{2}\right)+2 k(L, f)\right) .
$$

Proof. We set $D=a L+b f+D^{\prime}$, where $a, b \in \mathbb{Q}$ and $\left(D^{\prime}, L\right)=$ $\left(D^{\prime}, f\right)=0$. By the Hodge index theorem, $\left(D^{\prime 2}\right) \leq 0$. Hence $\left(D^{2}\right)=((a L+$ $\left.b f)^{2}\right)+\left(D^{\prime 2}\right) \leq\left((a L+b f)^{2}\right)=a^{2}\left(L^{2}\right)+2 a b(L, f)$. Thus we may assume that $D=a L+b f .(D, L+k f)=0$ implies that $b(L, f)=-a(L, L+k f)$. Hence $\left((a L+b f)^{2}\right)=-a^{2}\left(\left(L^{2}\right)+2 k(L, f)\right)$. Since $(L, f) \neq 0$, we get that $|a| \geq 1 /|(L, f)|$. Hence (1.1) holds.

Lemma 1.2. Let $r$ be a positive integer and $c_{1}$ an algebraically equivalence class on $X$ such that $\left(c_{1}, f\right)$ and $r$ are relatively prime. Let $L$ be an ample divisor on $X$. Then

$$
M_{L+n f}\left(r, c_{1}, \Delta\right)=\left\{\begin{array}{l}
E \mid \begin{array}{l}
E \text { is torsion free of rank } r \text { with }\left(c_{1}(E), \Delta(E)\right) \\
=\left(c_{1}, \Delta\right) \text { and }\left.E\right|_{\pi^{-1}(\eta)} \text { is stable. }
\end{array}
\end{array}\right\}
$$

for $n>\left(r^{3}(L, f)^{2} \Delta-2\left(L^{2}\right)\right) / 4(L, f)^{2}$. We denote this space by $M\left(r, c_{1}, \Delta\right)$.

Proof. The proof is similar to that in [Y3, Prop. 6.2] (in [Y3], we used slightly different definition of $\Delta$ ). 
Since $\operatorname{Ext}^{2}(E, E)_{0} \cong \operatorname{Hom}(E, E)_{0}^{\vee}=0, E \in M\left(r, c_{1}, \Delta\right), M\left(r, c_{1}, \Delta\right)$ is smooth of dimension $2 r \Delta-\left(r^{2}-1\right) \chi\left(\mathcal{O}_{X}\right)+\operatorname{dim} \operatorname{Pic}^{0}(X)$. For a stable sheaf $E \in M\left(r, c_{1}, \Delta\right), \chi\left(\left.E\right|_{f}\right)=\left(c_{1}, f\right)$ and $\chi\left(E \otimes k_{x}\right)=r$ are relatively prime, where $E$ is locally free at $x \in X$ and $k_{x}$ is the structure sheaf of $x$. Hence there is a universal family (cf. [Ma1, Thm. 6.11]).

LEMMA 1.3. Let $E$ be a vector bundle of rank $r$ on $X$ such that $\left(c_{1}(E), f\right)=d$, and let $F$ be a coherent sheaf of pure dimension 1 on a fibre $l$ with $\operatorname{rk}(F)=r_{1}$ and $\operatorname{deg}(F)=d_{1}$. Let $E \rightarrow F$ be a surjective homomorphism and $E^{\prime}$ the kernel. Then

$$
\Delta\left(E^{\prime}\right)=\Delta(E)+\frac{r d_{1}-r_{1} d}{r} .
$$

Proof. For a coherent sheaf $G$ on $X, \chi(G)=\operatorname{rk}(G) P\left(c_{1}(G) / \operatorname{rk} G\right)-$ $\Delta(G)$. Since $\chi(E)=\chi\left(E^{\prime}\right)+\chi(F)$,

$$
\begin{aligned}
\Delta\left(E^{\prime}\right)-\Delta(E) & =d_{1}-r\left(P\left(c_{1}(E) / \mathrm{rk} E\right)-P\left(c_{1}\left(E^{\prime}\right) / \mathrm{rk} E^{\prime}\right)\right) \\
& =d_{1}-\frac{r_{1} d}{r} .
\end{aligned}
$$

The following is a special case of Maruyama [Ma2].

Proposition 1.4. Let $E$ be a vector bundle on $X$ such that $\left.E\right|_{\pi^{-1}(\eta)}$ is a semi-stable vector bundle. Then there is a vector bundle $E^{\prime}$ on $X$ such that $\left.E^{\prime}\right|_{l}$ is semi-stable for every fibre $l$ and $E$ is obtained from $E^{\prime}$ by successive elementary transformations along coherent sheaves of pure dimension 1 on fibres.

Proof. We note that $\Delta(E) \geq 0$. We shall prove our claim by induction on $\Delta(E)$. We assume that there is a fibre $l$ such that $\left.E\right|_{l}$ is not semistable. Then there is a surjective homomorphism $\left.E\right|_{l} \rightarrow F$ such that $F$ is of pure dimension 1 and $\chi\left(\left.E\right|_{l}\right) / \operatorname{rk}\left(\left.E\right|_{l}\right)>\chi(F) / \operatorname{rk} F$. We shall consider the following elementary transformation along $F$ :

$$
0 \longrightarrow E_{1} \longrightarrow E \longrightarrow F \longrightarrow 0 \text {. }
$$

Since $\operatorname{depth}_{\mathcal{O}_{x}} F=1, x \in l$ and $X$ is smooth, we see that proj-dim $\operatorname{O}_{\mathcal{O}_{x}} F=$ $\operatorname{dim} X-\operatorname{depth}_{\mathcal{O}_{x}} F=1$. Hence $E_{1}$ is also locally free. By Lemma 1.3, we get that $\Delta\left(E_{1}\right)<\Delta(E)$. Hence we obtain our corollary. 


\subsection{General element of $M\left(r, c_{1}, \Delta\right)$}

If we fix the rank $r$ and the equivalence class $c_{1} \bmod \pi^{*} H^{1}(B, \mathbb{Z})$, then we may denote $M\left(r, c_{1}, \Delta\right)$ by $M(\Delta)$. In fact, $c_{1} \bmod r \pi^{*} H^{2}(B, \mathbb{Z})$ is determined by $r \Delta$ and the isomorphic class of $M_{H}\left(r, c_{1}, \Delta\right)$ is determined by $r$, $c_{1} \bmod r \pi^{*} H^{2}(B, \mathbb{Z})$ and $\Delta$.

Let $E$ be a general element of $M(\Delta)$. We shall consider the HarderNarasimhan filtration of the restriction $\left.E\right|_{l}$ of $E$ to fibres $l$. In particular, we shall show that $\left.E\right|_{l}$ is semi-stable for all singular fibres $l$.

LEMma 1.5. Let $C$ be a projective curve and $\mathcal{O}_{C}(1)$ an ample $d i$ visor on $C$. Let $L$ be a line bundle on $C$. Let $Q$ be the subscheme of

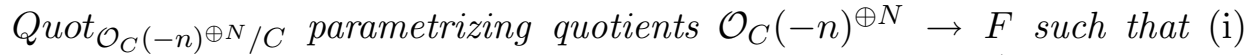
$F$ is a locally free sheaf of rank $r$ with $\operatorname{det} F=L$ and (ii) $H^{1}(C, F(n))=0$. Then $Q$ is smooth and irreducible.

Proof. Let $\lambda: \mathcal{O}_{C}(-n)^{\oplus N} \rightarrow F$ be a quotient which belongs to $Q$. Then we see that $\operatorname{Ext}^{1}(\operatorname{ker} \lambda, F)=0$. Since $\operatorname{Hom}(\operatorname{ker} \lambda, F) \rightarrow \operatorname{Ext}^{1}(F, F) \stackrel{\text { tr }}{\rightarrow}$ $H^{1}\left(C, \mathcal{O}_{C}\right)$ is surjective, $Q$ is smooth. For $k \geq n$, there is an exact sequence $0 \rightarrow \mathcal{O}_{C}^{\oplus(r-1)} \rightarrow F(k) \rightarrow L(r k) \rightarrow 0$. We set $\mathbb{P}:=\mathbb{P}\left(\operatorname{Ext}^{1}\left(L(r k), \mathcal{O}_{C}^{\oplus(r-1)}\right)^{\vee}\right)$. We shall consider the universal extension:

$$
0 \longrightarrow \mathcal{O}_{\mathbb{P} \times C}^{\oplus(r-1)} \longrightarrow \mathcal{F} \longrightarrow L(r k) \otimes \mathcal{O}_{\mathbb{P}}(-1) \longrightarrow 0 .
$$

Let $\mathbb{P}^{\prime}$ be the open subscheme of $\mathbb{P}$ of points $y$ such that $H^{1}\left(C, \mathcal{F}_{y}\right)=0$. Then $p_{\mathbb{P}^{\prime} *}(\mathcal{F})$ is a locally free sheaf on $\mathbb{P}^{\prime}$. Let $\phi: \mathbb{A} \rightarrow \mathbb{P}^{\prime}$ be the vector bundle associated to the locally free sheaf $\mathcal{H o m}\left(\mathcal{O}_{\mathbb{P}^{\prime}}^{\oplus N}, p_{\mathbb{P}^{\prime} *}(\mathcal{F})\right)$. Then there is a homomorphism $\Lambda: \mathcal{O}_{\mathbb{A} \times C}^{\oplus N} \rightarrow(\phi \times 1)^{*} \mathcal{F}$. Let $\mathbb{A}^{\prime}$ be the open subscheme of $\mathbb{A}$ such that $\Lambda$ is surjective. Then there is a surjective morphism $\mathbb{A}^{\prime} \rightarrow Q$, and hence $Q$ is irreducible.

Proposition 1.6. Let $M(\Delta)^{0}$ be the open subscheme of $M(\Delta)$ of elements $E$ such that $\left.E\right|_{l}$ is semi-stable for every singular fibre $l$. Then $M(\Delta)^{0}$ is a dense subscheme of $M(\Delta)$.

Proof. We note that $M(\Delta)_{0}:=M\left(r, c_{1}, \Delta\right)_{0}$ is an open dense subscheme of $M(\Delta)$ (cf. [Y1, Thm. 0.4]). Hence it is sufficient to prove that $M(\Delta)^{0} \cap M(\Delta)_{0}$ is an open dense subscheme of $M(\Delta)_{0}$. Let $E$ be a locally free stable sheaf of $M(\Delta)$. Since $\left.E\right|_{\pi^{-1}(\eta)}$ is stable, we see that $\operatorname{Ext}^{2}(E, E(-l))_{0} \cong$ $\operatorname{Hom}\left(E, E\left(l+K_{X}\right)\right)_{0}^{\vee}=0$. Hence we get that $\operatorname{Ext}^{1}(E, E)_{0} \rightarrow \operatorname{Ext}^{1}\left(\left.E\right|_{l},\left.E\right|_{l}\right)_{0}$ 
is surjective. Let $m$ be the multiplicity of $l$ and set $l=m l^{\prime}$. By Proposition 1.4, there is a vector bundle $E_{1}$ on $X$ such that $\left.E_{1}\right|_{l}$ is semi-stable and $\operatorname{det}\left(\left.E_{1}\right|_{l}\right)=\operatorname{det}\left(\left.E\right|_{l}\right) \otimes \mathcal{O}_{l}\left(k l^{\prime}\right)$. Since $\left(c_{1}, f\right)=m\left(c_{1}, l^{\prime}\right)$, our assumption on $r$ and $c_{1}$ implies that $(r, m)=1$. Replacing $E_{1}$ by $E_{1} \otimes \mathcal{O}_{X}\left(j l^{\prime}\right)$, we may assume that $\operatorname{det}\left(\left.E_{1}\right|_{l}\right)=\operatorname{det}\left(\left.E\right|_{l}\right)$. Let $\operatorname{Def}\left(\left.E\right|_{l}\right)$ be the local deformation space of $\left.E\right|_{l}$ of fixed determinant line bundle. We shall show that $\operatorname{Def}\left(\left.E\right|_{l}\right)^{u}:=\left\{F \in \operatorname{Def}\left(\left.E\right|_{l}\right) \mid F\right.$ is not semi-stable $\}$ is a proper closed subset of $\operatorname{Def}\left(\left.E\right|_{l}\right)$. In the notation of Lemma 1.5, we assume that $L=$ $\operatorname{det}\left(\left.E_{1}\right|_{l}\right)$ and $n$ is a sufficiently large integer such that there are quotients $\left.\mathcal{O}_{l}(-n)^{\oplus N} \rightarrow E\right|_{l},\left.\mathcal{O}_{l}(-n)^{\oplus N} \rightarrow E_{1}\right|_{l}$ which belong to $Q$. We also assume that $H^{0}\left(l, \mathcal{O}_{l}^{\oplus N}\right) \rightarrow H^{0}\left(l,\left.E\right|_{l}(n)\right)$ and $H^{0}\left(l, \mathcal{O}_{l}^{\oplus N}\right) \rightarrow H^{0}\left(l,\left.E_{1}\right|_{l}(n)\right)$ are isomorphisms. Let $\mathcal{O}_{Q \times l}(-n)^{\oplus N} \rightarrow \mathcal{Q}$ be the universal quotient. Since $\left.E_{1}\right|_{l}$ is semi-stable, Lemma 1.5 implies that $Q^{u}:=\left\{y \in Q \mid \mathcal{Q}_{y}\right.$ is not semi-stable $\}$ is a proper closed subset of $Q$. Since $\operatorname{Def}\left(\left.E\right|_{l}\right)$ is a transversal slice of the $\operatorname{Aut}\left(\mathcal{O}_{l}^{\oplus N}\right)$-orbit and $Q^{u}$ is $\operatorname{Aut}\left(\mathcal{O}_{l}^{\oplus N}\right)$-invariant, $\operatorname{Def}\left(\left.E\right|_{l}\right)^{u}$ is also a proper closed subset of $\operatorname{Def}\left(\left.E\right|_{l}\right)$. Combining the surjectivity of the homomorphism: $\operatorname{Ext}^{1}(E, E)_{0} \rightarrow \operatorname{Ext}^{1}\left(\left.E\right|_{l},\left.E\right|_{l}\right)_{0}$, we get that $M(\Delta)^{0}$ is an open dense subscheme of $M(\Delta)$.

Lemma 1.7. Let $l$ be a smooth fibre. Let $h:=\left\{\left(r_{1}, d_{1}\right), \ldots,\left(r_{s}, d_{s}\right)\right\}$ be a sequence of pairs of integers such that $r_{i}>0,1 \leq i \leq s$ and $d_{1} / r_{1}>$ $d_{2} / r_{2}>\cdots>d_{s} / r_{s}$. Let $D_{h}$ be the subset of $M\left(r, c_{1}, c_{2}\right)$ of elements $E$ such that the Harder-Narasimhan filtration of $\left.E\right|_{l}: 0 \subset F_{1} \subset F_{2} \subset \cdots \subset F_{s}=\left.E\right|_{l}$ satisfies that $\operatorname{rk}\left(F_{i} / F_{i-1}\right)=r_{i}$ and $\operatorname{deg}\left(F_{i} / F_{i-1}\right)=d_{i}, 1 \leq i \leq s$. Then $\operatorname{codim}\left(D_{h}\right) \geq \sum_{i<j} r_{j} d_{i}-r_{i} d_{j}$. In particular, if $\operatorname{codim}\left(D_{h}\right)=1$, then $s=2$ and $r_{2} d_{1}-r_{1} d_{2}=1$.

Proof. Let $\operatorname{Def}\left(\left.E\right|_{l}\right)_{h}$ be the subset of $\operatorname{Def}\left(\left.E\right|_{l}\right)$ of elements $G$ such that the Harder-Narasimhan filtration of $G: 0 \subset F_{1} \subset F_{2} \subset \cdots \subset F_{s}=G$ satisfies that $\operatorname{rk}\left(F_{i} / F_{i-1}\right)=r_{i}$ and $\operatorname{deg}\left(F_{i} / F_{i-1}\right)=d_{i}, 1 \leq i \leq s$. We assume that $\operatorname{Def}\left(\left.E\right|_{l}\right)_{h}$ is not empty. We note that $\operatorname{Ext}^{1}(E, E)_{0} \rightarrow \operatorname{Ext}^{1}\left(\left.E\right|_{l},\left.E\right|_{l}\right)_{0}$ is surjective. It is known that $\operatorname{codim}\left(\operatorname{Def}\left(\left.E\right|_{l}\right)_{h}\right)=\sum_{i<j} r_{j} d_{i}-r_{i} d_{j}$ (cf. [A-B, Thm. 7.14]). Hence we get our lemma.

Let $\left(r_{1}, d_{1}\right)$ be the pair of integers such that $0<r_{1}<r$ and $r d_{1}-r_{1} d=1$. Let $M(\Delta)^{1}$ be the open subscheme of $M(\Delta)^{0}$ of elements $E$ such that $\left.E\right|_{l}$ is stable, or the Harder-Narasimhan filtration of $\left.E\right|_{l}$ is $\left.0 \subset F \subset E\right|_{l}$ for every smooth fibre $l$, where $F$ is a stable vector bundle of rank $r_{1}$ on $l$ with $\operatorname{deg}(F)=d_{1}$. Then $M(\Delta)^{1}$ is an open dense subscheme of $M(\Delta)^{0}$. 


\subsection{Vector bundles on elliptic curves}

The following is due to Atiyah [A].

Lemma 1.8. Let $C$ be a smooth elliptic curve. Let $r$ be a positive integer and $d$ an integer such that $(r, d)=1$. Then,

(1) There is a stable vector bundle of rank $r$ and degree $d$.

(2) Let $\left(r_{1}, d_{1}\right)$ be the pair of integers such that $r_{1} d-r d_{1}=1$ and $0<r_{1}<r$. Let $E_{1}$ be a stable vector bundle of rank $r_{1}$ and degree $d_{1}$. Then every stable vector bundle $E$ of rank $r$ and degree $d$ is defined by an exact sequence

$$
0 \longrightarrow E_{1} \longrightarrow E \longrightarrow E_{2} \longrightarrow 0
$$

where $E_{2}$ is a stable vector bundle of rank $r_{2}:=r-r_{1}$ and degree $d_{2}:=d-d_{1}$. (3) Let $0 \subset F_{1} \subset F_{2} \subset \cdots \subset F_{s}=E$ be the Harder-Narasimhan filtration of a vector bundle $E$. Then $E \cong \bigoplus_{i=1}^{s} E_{i}$, where $E_{i}:=F_{i} / F_{i-1}$.

Proof. (1) We shall prove our claim by induction on $r$. If $r=1$, then our claim obviously holds. Let $\left(r_{1}, d_{1}\right)$ be the pair of integers such that $r_{1} d-r d_{1}=1$ and $0<r_{1}<r$. We set $r_{2}:=r-r_{1}$ and $d_{2}:=d-d_{1}$. By induction hypothesis, there are stable vector bundles $E_{i}$ of rank $r_{i}$ and degree $d_{i}, i=1,2$. Since $d_{1} / r_{1}<d_{2} / r_{2}, \operatorname{Hom}\left(E_{2}, E_{1}\right)=0$. By using the Riemann-Roch theorem, we get that $\operatorname{Ext}^{1}\left(E_{2}, E_{1}\right) \cong \mathbb{C}$. Let $0 \rightarrow E_{1} \rightarrow$ $E \rightarrow E_{2} \rightarrow 0$ be a non-trivial extension. We shall show that $E$ is stable. If $E$ is not stable, then there is a semi-stable subsheaf $G$ of $E$ such that $\operatorname{deg} G / \operatorname{rk} G>d / r$. Since $G$ and $E_{2}$ are semi-stable and $G \rightarrow E \rightarrow E_{2}$ is not zero, $\operatorname{deg} G / \operatorname{rk} G \leq d_{2} / r_{2}$. We assume that $\operatorname{deg} G / \operatorname{rk} G<d_{2} / r_{2}$. Then we see that $1 / r r_{2}=d_{2} / r_{2}-d / r>d_{2} / r_{2}-\operatorname{deg} G / \operatorname{rk} G \geq 1 / r_{2} \operatorname{rk} G$, which is a contradiction. Hence $\operatorname{deg} G / \operatorname{rk} G=d_{2} / r_{2}$. Then we get that $\operatorname{rk} G=r_{2}$ and $\operatorname{deg} G=d_{2}$. Hence $G \cong E_{2}$, which is a contradiction.

(2) Let $E$ be a stable vector bundle of rank $r$ and degree $d$. Then $\operatorname{Ext}^{1}\left(E_{1}, E\right) \cong \operatorname{Hom}\left(E, E_{1}\right)^{\vee}=0$. By the Riemann-Roch theorem, there is a non-zero homomorphism $\varphi: E_{1} \rightarrow E$. We shall show that $\varphi$ is injective and coker $\varphi$ is stable. Since $E_{1}$ and $E$ are stable, $d_{1} / r_{1} \leq \operatorname{deg} \varphi\left(E_{1}\right) / \operatorname{rk} \varphi\left(E_{1}\right)<$ $d / r$. In the same way as in the proof of $(1)$, we see that $\operatorname{rk} \varphi\left(E_{1}\right)=r_{1}$ and $\operatorname{deg} \varphi\left(E_{1}\right)=d_{1}$. Hence we get that $E_{1} \cong \varphi\left(E_{1}\right)$. We set $E_{2}:=\operatorname{coker} \varphi$. We assume that there is a quotient $G$ of $E_{2}$ such that $G$ is semi-stable and $d_{2} / r_{2}>\operatorname{deg} G / \operatorname{rk} G$. Since $G$ is a quotient of $E$, we get that $d / r<$ $\operatorname{deg} G / \operatorname{rk} G$. Hence we get that $d / r<\operatorname{deg} G / \operatorname{rk} G<d_{2} / r_{2}$. Then $1 / r r_{2}=$ 
$d_{2} / r_{2}-d / r>d_{2} / r_{2}-\operatorname{deg} G / \operatorname{rk} G \geq 1 / r_{2} \operatorname{rk} G$, which is a contradiction. Hence $E_{2}$ is a stable vector bundle.

(3) Since $\operatorname{deg} E_{i} / \mathrm{rk} E_{i}>\operatorname{deg} E_{j} / \mathrm{rk} E_{j}, i<j$, the Serre duality implies that $\operatorname{Ext}^{1}\left(E_{j}, E_{i}\right)=0, i<j$. By the induction on $s$, we see that $E \cong \bigoplus_{i} E_{i}$.

Lemma 1.9. Let $(r, d)$ (resp. $\left.\left(r_{1}, d_{1}\right),\left(r_{2}, d_{2}\right)\right)$ be the pair in Lemma 1.8 . Let $E$ be a vector bundle of rank $r$ on an elliptic curve $C$ with degree $d$ and $E_{2}$ a stable vector bundle of rank $r_{2}$ on $C$ with degree $d_{2}$.

(1) If $E$ is stable, then $\operatorname{Hom}\left(E, E_{2}\right) \cong \mathbb{C}$ and a non-zero homomorphism is surjective.

(2) Let $F_{1}$ (resp. $F_{2}$ ) be a stable vector bundle of rank $r_{1}$ and degree $d_{1}$ (resp. rank $r_{2}$ and degree $\left.d_{2}\right)$. We assume that $E \cong F_{1} \oplus F_{2}$ and there is a surjective homomorphism $\varphi: E \rightarrow E_{2}$ such that $\operatorname{ker} \varphi$ is also stable. Then $E_{2} \cong F_{2}$ and $\operatorname{Hom}\left(E, E_{2}\right) \cong \mathbb{C}^{\oplus 2}$.

Proof. (1) Since $E$ is stable, $\operatorname{Ext}^{1}\left(E, E_{2}\right) \cong \operatorname{Hom}\left(E_{2}, E\right)^{\vee}=0$. By the Riemann-Roch theorem, we see that $\operatorname{dim} \operatorname{Hom}\left(E, E_{2}\right)=1$. In the same way as in the proof of Lemma 1.8, we see that a non-zero homomorphism $E \rightarrow E_{2}$ is surjective.

(2) If $E_{2} \neq F_{2}$, then $\operatorname{ker} \varphi \cong \operatorname{ker}\left(\left.\varphi\right|_{F_{1}}\right) \oplus F_{2}$. Since $\left.\varphi\right|_{F_{1}}: F_{1} \rightarrow E_{2}$ is surjective, $\operatorname{ker}\left(\left.\varphi\right|_{F_{1}}\right) \neq 0$. Hence $E_{2} \cong F_{2}$. By the Riemann-Roch theorem, $\operatorname{Hom}\left(F_{1}, E_{2}\right) \cong \mathbb{C}$. Therefore $\operatorname{Hom}\left(E, E_{2}\right) \cong \mathbb{C}^{\oplus 2}$.

\section{4 .}

Let $B_{0}$ be the open subscheme of $B$ such that $\pi: X_{0}:=X \times_{B} B_{0} \rightarrow$ $B_{0}$ is smooth. We assume that $\pi$ has a section $\sigma$. We denote the relative moduli space of stable vector bundles of rank $r$ on fibres with degree $d$ by $\mathcal{M}_{X_{0} / B_{0}}(r, d) \rightarrow B_{0}$. We assume that $(r, d)=1$. We shall construct a family of stable vector bundles $\mathcal{E}_{r, d}$ on $X_{0} \times{ }_{B_{0}} X_{0}$ and show that $\mathcal{M}_{X_{0} / B_{0}}(r, d) \cong X_{0}$ as a $B_{0}$-scheme, by using induction on $r$. If $r=1$, then $\mathcal{E}_{1, d}:=\mathcal{O}_{X_{0} \times{ }_{B_{0}} X_{0}}((d+1) \sigma-\Delta)$ is a universal family, where $\Delta$ is the diagonal of $X_{0} \times{ }_{B_{0}} X_{0}$. Let $\left(r_{1}, d_{1}\right)$ be the pair of integers such that $r_{1} d-r d_{1}=1$ and $0<r_{1}<r$. We set $r_{2}=r-r_{1}$ and $d_{2}=d-d_{1}$. Let $E$ be a vector bundle on $X_{0}$ such that $\left.E\right|_{l}$ is a stable vector bundle of rank $r_{2}$ and $\left.\operatorname{det} E\right|_{l} \cong \mathcal{O}_{l}\left(d_{2} \sigma\right)$ for every fibre $l$. By using Lemma 1.8 , we see that $\mathcal{L}:=\operatorname{Ext}_{p_{X_{0}}}^{1}\left(E, \mathcal{E}_{r_{1}, d_{1}}\right)$ is a line bundle on $X_{0}$. Then there is the universal extension

$$
0 \longrightarrow \mathcal{E}_{r_{1}, d_{1}} \longrightarrow \mathcal{E}_{r, d} \longrightarrow E \otimes p_{X_{0}}^{*}(\mathcal{L}) \longrightarrow 0
$$


which parametrizes stable vector bundles of rank $r$ on fibres with degree $d$. Hence there is a morphism $X_{0} \rightarrow \mathcal{M}_{X_{0} / B_{0}}(r, d)$. By our construction, this morphism is injective. By ZMT, it is an isomorphism.

LEMma 1.10. Let $E$ and $E^{\prime}$ be semi-stable vector bundles on a multiple fibre $l=m l^{\prime}$ such that $\mathrm{rk} E=\mathrm{rk} E^{\prime}, \operatorname{det} E \cong \operatorname{det} E^{\prime}$, and $\chi(E)=\chi\left(E^{\prime}\right)=d$. Then,

$$
\operatorname{Hom}\left(E, E^{\prime}\right)= \begin{cases}\mathbb{C}, & \text { if } E \cong E^{\prime} \\ 0, & \text { otherwise. }\end{cases}
$$

Proof. We set $L:=\left.\mathcal{O}_{X}\left(-l^{\prime}\right)\right|_{l^{\prime}}$. We note that $\operatorname{rk}\left(E \otimes L^{\otimes k}\right)=r$ and $\chi\left(E \otimes L^{\otimes k}\right)=d / m$ for $0 \leq k \leq m-1$. Since $(r, d)=1$ and $E$ is semi-stable, $E \otimes L^{\otimes k}$ is a stable sheaf on $m l^{\prime}$. Thus $0 \subset E\left(-(m-1) l^{\prime}\right) \subset E\left(-(m-2) l^{\prime}\right) \subset$ $\cdots \subset E\left(-l^{\prime}\right) \subset E$ is a Jordan-Hölder filtration of $E$. Since the order of $L \in \operatorname{Pic}^{0}\left(l^{\prime}\right)$ is $m$ and $(m, r)=1$, $\operatorname{det} E \cong \operatorname{det} E^{\prime}$ and the stabilities of $\left.E\right|_{l^{\prime}}$ and $\left.E\right|_{l^{\prime}}$ imply that $\operatorname{Hom}\left(\left.E\right|_{l}, E^{\prime} \otimes L^{\otimes k}\right)=0$ for $1 \leq k \leq m-1$. Let $\varphi: E \rightarrow E^{\prime}$ be a non-zero homomorphism. We shall show that $\varphi$ is an isomorphism. Since $\operatorname{Hom}\left(\left.E\right|_{l}, E^{\prime} \otimes L^{\otimes k}\right)=0$ for $1 \leq k \leq m-1$, we see that $\left.\varphi\right|_{l^{\prime}}:\left.\left.E\right|_{l^{\prime}} \rightarrow E^{\prime}\right|_{l^{\prime}}$ is not zero, which implies that $\left.\left.E\right|_{l^{\prime}} \cong E^{\prime}\right|_{l^{\prime}}$. By Nakayama's lemma, $\varphi$ is an isomorphism. Then it is easy to see that $\operatorname{Hom}\left(E, E^{\prime}\right) \cong \mathbb{C}$.

Lemma 1.11. Let $E, E^{\prime}$ be vector bundles of rank $r$ on $X$ such that $\left.E\right|_{l}$ and $\left.E^{\prime}\right|_{l}$ are semi-stable for all fibres $l$ and $\operatorname{det} E \cong \operatorname{det} E^{\prime}$. Then there is a line bundle $L$ on $B$ such that $E \cong E^{\prime} \otimes \pi^{*}(L)$.

Proof. We note that $\left.\left.E\right|_{\pi^{-1}(\eta)} \cong E^{\prime}\right|_{\pi^{-1}(\eta)}$. By the upper semi-continuity of $h^{0}\left(l,\left.E^{\prime \vee} \otimes E\right|_{l}\right)$, there is a non-zero homomorphism $\left.\left.E^{\prime}\right|_{l} \rightarrow E\right|_{l}$ for every fibre $l$. Since $\left.E\right|_{l}$ and $\left.E^{\prime}\right|_{l}$ are semi-stable, Lemma 1.10 implies that $\left.E\right|_{l} \cong$ $\left.E^{\prime}\right|_{l}$ and $H^{0}\left(l,\left.E^{\prime} \vee \otimes E\right|_{l}\right) \cong \mathbb{C}$. By the base change theorem, we get that $L:=\pi_{*}\left(E^{\prime \vee} \otimes E\right)$ is a line on $B$ and $\pi^{*}(L) \otimes E^{\prime} \rightarrow E$ is an isomorphism.

Corollary 1.12. $M(\Delta)$ is not empty if and only if $\Delta \geq \Delta_{0}:=$ $\frac{\left(r^{2}-1\right)}{2 r} \chi\left(\mathcal{O}_{X}\right)$.

Proof. We set $\Delta^{\prime}:=\min \{\Delta \mid M(\Delta) \neq \emptyset\}$. Lemma 1.11 implies that $\operatorname{dim} \operatorname{Pic}^{0}(X)=\operatorname{dim} M\left(\Delta^{\prime}\right)=2 r \Delta^{\prime}-\left(r^{2}-1\right) \chi\left(\mathcal{O}_{X}\right)+\operatorname{dim} \operatorname{Pic}^{0}(X)$. Hence we get our claim. 
Remark 1.1. Let $E$ be an element of $M\left(\Delta_{0}\right)$. By Lemma 1.11, there is a surjective morphism $\operatorname{Pic}^{0}(X) \rightarrow M\left(\Delta_{0}\right)$ sending $L \in \operatorname{Pic}^{0}(X)$ to $E \otimes L$. Hence we get that $M\left(\Delta_{0}\right)=\operatorname{Pic}^{0}(X) / \Phi(E)$, where $\Phi(E):=\left\{L \in \operatorname{Pic}^{0}(X) \mid\right.$ $E \otimes L \cong E\}$. In particular, if $\operatorname{Pic}^{0}(X)=\operatorname{Pic}^{0}(B)$, then $M\left(\Delta_{0}\right)=\operatorname{Pic}^{0}(X)$.

\subsection{Construction of a family}

We assume that $\pi: X \rightarrow B$ has a section and show that $M(\Delta)$ is birational to $M\left(\Delta_{0}\right) \times S^{n} X$, where $n:=r\left(\Delta-\Delta_{0}\right)$. Let $\mathcal{E}$ be a universal family on $M\left(\Delta_{0}\right) \times X$. Let $\left(r_{1}, d_{1}\right)$ be the pair of integers such that $r_{1} d-$ $r d_{1}=-1$ and $0<r_{1}<r$, and let $\mathcal{E}_{r_{1}, d_{1}}$ be the vector bundle on $X_{0} \times_{B_{0}} X_{0}$. Let $j: X_{0} \times{ }_{B_{0}} X_{0} \rightarrow X_{0} \times X$ be the immersion. We denote the projection $M\left(\Delta_{0}\right) \times X_{0} \rightarrow M\left(\Delta_{0}\right)$ by $q_{1}$ and $M\left(\Delta_{0}\right) \times X_{0} \rightarrow X_{0}$ by $q_{2}$. By Lemma 1.9, $\mathcal{L}:=\operatorname{Hom}_{p_{M\left(\Delta_{0}\right) \times X_{0}}}\left(\left(q_{1} \times 1_{X}\right)^{*} \mathcal{E},\left(q_{2} \times 1_{X}\right)^{*} j_{*} \mathcal{E}_{r_{1}, d_{1}}\right)$ is a line bundle on $M\left(\Delta_{0}\right) \times X_{0}$, and there is a surjective homomorphism: $\left(q_{1} \times 1_{X}\right)^{*} \mathcal{E} \rightarrow$ $\left(q_{2} \times 1_{X}\right)^{*} j_{*} \mathcal{E}_{r_{1}, d_{1}} \otimes p_{M\left(\Delta_{0}\right) \times X_{0}}^{*}(\mathcal{L})^{\vee}$. Let $p_{i}: X_{0}^{n}:=X_{0} \times X_{0} \times \cdots \times X_{0} \rightarrow X_{0}$ be the $i$-th projection, $1 \leq i \leq n$. Then there is a homomorphism

$$
\Lambda: \widetilde{\mathcal{E}} \longrightarrow \bigoplus_{i=1}^{n}\left(q_{2} \circ\left(1_{M\left(\Delta_{0}\right)} \times p_{i}\right) \times 1_{X}\right)^{*} j_{*} \mathcal{E}_{r_{1}, d_{1}} \otimes \mathcal{L}_{i}
$$

where $\widetilde{\mathcal{E}}$ is the pull-back of $\mathcal{E}$ to $M\left(\Delta_{0}\right) \times X_{0}^{n} \times X$ and $\mathcal{L}_{i}=\left(1_{M\left(\Delta_{0}\right)} \times\right.$ $\left.p_{i} \times 1_{X}\right)^{*} p_{M\left(\Delta_{0}\right) \times X_{0}}^{*}(\mathcal{L})^{\vee}$. We set $\Gamma:=\left\{\left(x_{1}, x_{1}, \ldots, x_{n}\right) \in X_{0}^{n} \mid \pi\left(x_{i}\right)=\right.$ $\pi\left(x_{j}\right)$ for some $\left.i \neq j\right\}$. Then $\Lambda_{1}:=\left.\Lambda\right|_{M\left(\Delta_{0}\right) \times\left(X_{0}^{n} \backslash \Gamma\right) \times X}$ is a surjective homomorphism. We set $\mathcal{F}:=\operatorname{ker} \Lambda_{1}$. By Lemma $1.3, \mathcal{F}$ is a family of stable vector bundles on $X$. Hence there is a morphism $M\left(\Delta_{0}\right) \times\left(X_{0}^{n} \backslash \Gamma\right) \rightarrow M(\Delta)$. By our construction, this morphism is $\mathfrak{S}_{n}$-invariant, and hence we get a morphism $\nu: M\left(\Delta_{0}\right) \times\left(X_{0}^{n} / \mathfrak{S}_{n}\right) \rightarrow M(\Delta)$. By our construction, it is injective. Since $\operatorname{dim} S^{n} X=2 n=\operatorname{dim} M(\Delta)-\operatorname{dim} M\left(\Delta_{0}\right)$, ZMT implies that $M\left(\Delta_{0}\right) \times\left(X_{0}^{n} / \mathfrak{S}_{n}\right) \rightarrow M(\Delta)$ is an immersion. We set $M(\Delta)^{2}:=$ $\nu\left(M\left(\Delta_{0}\right) \times\left(X_{0}^{n} / \mathfrak{S}_{n}\right)\right)$. We shall show that $M(\Delta)^{2}$ is dense. For this purpose, we shall estimate the dimension of $M(\Delta)^{1} \backslash M(\Delta)^{2}$.

Lemma 1.13. $\operatorname{dim}\left(M(\Delta)^{1} \backslash M(\Delta)^{2}\right)=2 n-1+\operatorname{dim} M\left(\Delta_{0}\right)$.

Proof. Assume that the restriction $\left.E\right|_{l}$ of $E \in M(\Delta)^{1}$ to a smooth fibre $l$ is not stable. By the definition of $M(\Delta)^{1}$, we see that $\left.E\right|_{l} \cong E_{1} \oplus E_{2}$, where $E_{1}$ (resp. $E_{2}$ ) is a stable vector bundle of rank $r_{1}$ and degree $d_{1}$ (resp. rank $r_{2}$ and degree $\left.d_{2}\right)$. We set $E^{\prime}:=\operatorname{ker}\left(E \rightarrow E_{1}\right)$. Then there is an exact sequence

$$
\left.0 \longrightarrow E_{1} \longrightarrow E^{\prime}\right|_{l} \longrightarrow E_{2} \longrightarrow 0
$$


Then $E$ is obtained by the inverse transform from $E^{\prime}$ :

$$
0 \longrightarrow E \longrightarrow E^{\prime}(l) \longrightarrow E_{2} \longrightarrow 0 .
$$

By (1.7), $\left.E^{\prime}\right|_{l}$ is stable or $\left.E^{\prime}\right|_{l} \cong E_{1} \oplus E_{2}$. By Lemma 1.3, $\Delta\left(E^{\prime}\right)=$ $\Delta(E)-1 / r$. Conversely, for $E^{\prime} \in M(\Delta-1 / r)^{1}$, we shall consider a surjective homomorphism $\psi: E^{\prime} \rightarrow F_{2}$ such that the kernel of $\left.E^{\prime}\right|_{l} \rightarrow F_{2}$ is stable, where $F_{2}$ is a stable vector bundle of rank $r_{2}$ on a smooth fibre $l$ with degree $d_{2}$. If ker $\psi \otimes \mathcal{O}_{X}(l)$ belongs to $M(\Delta)^{1} \backslash M(\Delta)^{2}$, then (i) $\left.E^{\prime}\right|_{l}$ is stable and $E^{\prime}$ belongs to $M(\Delta-1 / r)^{1} \backslash M(\Delta-1 / r)^{2}$, or (ii) $\left.E^{\prime}\right|_{l}$ is not stable and $F_{2}$ is a direct summand of $\left.E^{\prime}\right|_{l}$. Since $\#\left\{l\left|E^{\prime}\right|_{l}\right.$ is not stable $\} \leq n-1$, by using Lemma 1.9, we see that

$$
\begin{aligned}
& \operatorname{dim}\left(M(\Delta)^{1} \backslash M(\Delta)^{2}\right) \\
& \quad=\max \left\{\operatorname{dim}\left(M(\Delta-1 / r)^{1} \backslash M(\Delta-1 / r)^{2}\right)+2, \operatorname{dim} M(\Delta-1 / r)^{1}+1\right\} \\
& \quad=2 n-1+\operatorname{dim} M\left(\Delta_{0}\right) .
\end{aligned}
$$

THEOREM 1.14. $M(\Delta)$ is irreducible and birational to $M\left(\Delta_{0}\right) \times$ $S^{n}\left(J^{d_{1}} X\right)$, where $n:=r\left(\Delta-\Delta_{0}\right)$.

Proof. If $\pi: X \rightarrow B$ has a section, we have proved our theorem. For general cases, we shall consider a Galois covering $\gamma: B^{\prime} \rightarrow B$ such that $\pi^{\prime}: X \times_{B} B^{\prime} \rightarrow B^{\prime}$ has a section $\sigma^{\prime}$. Let $B_{1}$ be an open subscheme of $B_{0}$ such that $\gamma^{-1}\left(B_{1}\right) \rightarrow B_{1}$ is etale. We set $X_{1}^{\prime}:=\pi^{-1}\left(B_{1}\right) \times_{B} B^{\prime}$. Let $\mathcal{E}_{r_{1}, d_{1}}^{\prime}$ be the vector bundle on $X_{1}^{\prime} \times_{\gamma^{-1}\left(B_{1}\right)} X_{1}^{\prime}$ and $j^{\prime}: X_{1}^{\prime} \times_{\gamma^{-1}\left(B_{1}\right)} X_{1}^{\prime} \cong X_{1}^{\prime} \times_{B_{1}}$ $X_{1} \hookrightarrow X_{1}^{\prime} \times X_{1}$ the inclusion. Let $X_{1}^{\prime} \rightarrow J^{d_{1}} X$ be the morphism induced by $\mathcal{E}_{r_{1}, d_{1}}^{\prime}$. For a $g \in \operatorname{Gal}\left(B^{\prime} / B\right)$, let $\tilde{g}: X_{1}^{\prime} \rightarrow X_{1}^{\prime}$ be the automorphism of $X_{1}^{\prime}$ sending $(x, y) \in \pi^{-1}\left(B_{1}\right) \times_{B} B^{\prime}$ to $\left(x+\left(d_{1}-1\right)\left(\sigma^{\prime}(g(y))-\sigma^{\prime}(y)\right), g(y)\right)$. Then it defines an action of $\operatorname{Gal}\left(B^{\prime} / B\right)$ to $X_{1}^{\prime}$. By the construction of $\mathcal{E}_{r_{1}, d_{1}}^{\prime}$, we see that $\left.\left.\operatorname{det}\left(\mathcal{E}_{r_{1}, d_{1}}^{\prime}\right)\right|_{\tilde{g}((x, y))} \cong \operatorname{det}\left(\mathcal{E}_{r_{1}, d_{1}}^{\prime}\right)\right|_{(x, y)}$. Hence $\left.\left(\mathcal{E}_{r_{1}, d_{1}}^{\prime}\right)\right|_{\tilde{g}((x, y))} \cong$ $\left.\left(\mathcal{E}_{r_{1}, d_{1}}^{\prime}\right)\right|_{(x, y)}$. Thus the morphism $X_{1}^{\prime} \rightarrow J^{d_{1}} X$ is $\operatorname{Gal}\left(B^{\prime} / B\right)$-invariant. Then we get that $X_{1}^{\prime} / \operatorname{Gal}\left(B^{\prime} / B\right) \rightarrow J^{d_{1}} X$ is an immersion. Replacing $j_{*} \mathcal{E}_{r_{1}, d_{1}}$ by $j_{*}^{\prime} \mathcal{E}_{r_{1}, d_{1}}^{\prime}$, we can construct a family of stable vector bundles $\mathcal{F}$ parametrized by $M\left(\Delta_{0}\right) \times\left(\left(X_{1}^{\prime}\right)^{n} \backslash \Gamma^{\prime}\right)$, where $\Gamma^{\prime}$ is the pull-back of $\Gamma$ to $\left(X_{1}^{\prime}\right)^{n}$. Hence we get a morphism $M\left(\Delta_{0}\right) \times\left(\left(X_{1}^{\prime}\right)^{n} \backslash \Gamma^{\prime}\right) \rightarrow M(\Delta)$. By the construction, $\operatorname{Gal}\left(B^{\prime} / B\right) \times \mathfrak{S}_{n}$ acts on $\left(\left(X_{1}^{\prime}\right)^{n} \backslash \Gamma^{\prime}\right)$, and this morphism is $\operatorname{Gal}\left(B^{\prime} / B\right) \times \mathfrak{S}_{n^{-}}$ invariant. Hence we get a morphism $M\left(\Delta_{0}\right) \times\left(\left(J^{d_{1}} X_{1}\right)^{n} \backslash \Gamma\right) / \mathfrak{S}_{n} \rightarrow M(\Delta)$. Then we see that $M(\Delta)$ is birationally equivalent to $M\left(\Delta_{0}\right) \times S^{n}\left(J^{d_{1}} X\right)$. 


\section{$\S 2$. Moduli spaces on Del Pezzo surfaces}

\section{1 .}

We shall apply Theorem 1.14 to moduli spaces on Del Pezzo surfaces.

ThEOREM 2.1. We assume that $X=\mathbb{P}^{2}$ and set $H:=\mathcal{O}_{\mathbb{P}^{2}}(1)$. Then $M_{H}(r, k H, \Delta)$ is a rational variety if $(r, 3 k)=1$.

Proof. Let $V \subset H^{0}\left(\mathbb{P}^{2}, \mathcal{O}_{\mathbb{P}^{2}}(3)\right)$ be a pencil such that every member $D \in V$ is irreducible and $\#\left\{P \mid P \in \bigcap_{D \in V} D\right\}=9$. Let $\phi: Y \rightarrow \mathbb{P}^{2}$ be the blow-ups of $\mathbb{P}^{2}$ at base points of $V$. Then there is an elliptic fibration $\pi: Y \rightarrow \mathbb{P}^{1}$ such that every fibre is isomorphic to a member $D$ of $V$. We set

$$
N:=\left\{E \in M_{H}(r, k H, \Delta)_{0}\left|\phi^{*} E\right|_{\pi^{-1}(\eta)} \text { is stable }\right\}
$$

where $\eta$ is the generic point of $\mathbb{P}^{1}$. Let $E$ be a stable vector bundle of rank $r$ on $\mathbb{P}^{2}$ with $c_{1}(E)=k H$. Then $\operatorname{Ext}^{2}(E, E(-3))_{0} \cong \operatorname{Hom}(E, E)_{0}^{\vee}=0$. Let $D \in V$ be a smooth elliptic curve. Then we get the surjective homomorphism $\operatorname{Ext}^{1}(E, E)_{0} \rightarrow \operatorname{Ext}^{1}\left(\left.E\right|_{D},\left.E\right|_{D}\right)_{0}$. Hence $\operatorname{Def}(E) \rightarrow \operatorname{Def}\left(\left.E\right|_{D}\right)$ is submersive. Since $\left(r, \operatorname{deg}\left(\left.E\right|_{D}\right)\right)=(r, 3 k)=1$, we can deform $E$ to a stable sheaf $F$ such that $\left.F\right|_{D}$ is a stable vector bundle on $D$. By the openness of stability, $\left.F\right|_{\pi^{-1}(\eta)}$ is a stable vector bundle. Hence $N$ is an open dense subscheme of $M_{H}(r, k H, \Delta)$ and there is an open immersion $\phi^{*}: N \rightarrow M\left(r, k \phi^{*} H, \Delta\right)$. By Theorem 1.14, $N$ is bitarional to $S^{n} Y$, where $n=r \Delta-\left(r^{2}-1\right) / 2$. Since $S^{n} Y$ is a rational variety, we get our theorem.

DEFINITION 2.1. $\operatorname{Spl}\left(r, c_{1}, \Delta\right)$ is the moduli space of simple torsion free sheaves $E$ of rank $r$ with $c_{1}(E)=c_{1}$ and $\Delta(E)=\Delta$.

We shall next consider the irreducibility of $\operatorname{Spl}\left(r, c_{1}, \Delta\right)$ for Del Pezzo surfaces.

Proposition 2.2. Let $\pi: X \rightarrow \mathbb{P}^{1}$ be a rational elliptic surface with a section $\sigma$. For a divisor class $c_{1} \in \mathrm{NS}(X)$ such that $\left(c_{1}, f\right)$ and $r$ are relatively prime, we shall consider the moduli space $M(\Delta)=M\left(r, c_{1}, \Delta\right)$. Then $M(\Delta)$ is irreducible and rational.

Proof. Since $\pi$ has a section $\sigma$, the canonical bundle formula implies that $K_{X} \cong \pi^{*} \mathcal{O}_{\mathbb{P}^{1}}(-1)$. Hence $\sigma$ is an exceptional curve of the first kind by the adjunction formula $\left(K_{X}+\sigma, \sigma\right)=-2$. Since $R^{1} \pi_{*} \mathcal{O}_{X}$ is locally free 
of rank 1 , the exact sequence $0 \rightarrow \mathcal{O}_{X} \rightarrow \mathcal{O}_{X}(\sigma) \rightarrow \mathcal{O}_{\sigma}(-1) \rightarrow 0 \mathrm{im}$ plies that $\pi_{*} \mathcal{O}_{\sigma}(-1) \cong R^{1} \pi_{*} \mathcal{O}_{X}$, and hence we get that $\pi_{*} K_{X}^{\vee}(\sigma) \cong \pi_{*} K_{X}^{\vee}$. Let $\phi: X \rightarrow Y$ be the contraction of $\sigma$. Then we get that $H^{0}\left(Y, K_{Y}^{\vee}\right) \cong$ $H^{0}\left(X, K_{X}^{\vee}(\sigma)\right) \cong H^{0}\left(X, K_{X}^{\vee}\right) \cong \mathbb{C}^{\oplus 2}$. By the Riemann-Roch theorem, $H^{1}\left(Y, K_{Y}^{\vee}\right)=0$. Let $\delta: \mathcal{Y} \rightarrow S$ be a smooth family of 8-points blow-ups of $\mathbb{P}^{2}$ such that $H^{1}\left(\mathcal{Y}_{s}, K_{\mathcal{Y}_{s}}^{\vee}\right)=0$ for all $s \in S$ and $\mathcal{Y}_{s_{0}}=Y$ for some $s_{0} \in S$. Let $\xi$ be the generic point of $S$. By the base change theorem, $\delta_{*}\left(K_{\mathcal{Y} / S}^{\vee}\right)$ is a locally free sheaf of rank 2 and $\delta_{*}\left(K_{\mathcal{Y} / S}^{\vee}\right) \otimes k(s) \rightarrow H^{0}\left(K_{Y_{s}}^{\vee}\right), s \in S$ is an isomorphism. We set $\mathcal{O}_{\mathcal{Z}}:=\operatorname{coker}\left(\delta^{*} \delta_{*}\left(K_{\mathcal{Y} / S}^{\vee}\right) \rightarrow K_{\mathcal{Y} / S}^{\vee}\right) \otimes K_{\mathcal{Y} / S}$. Then $\mathcal{O}_{\mathcal{Z}} \otimes k(s)$ defines a reduced one point of $Y_{s}$. Thus $\mathcal{Z}$ defines a section of $\delta$. Let $\phi_{S}: \mathcal{X} \rightarrow \mathcal{Y}$ be the blow-up of $\mathcal{Y}$ along $\mathcal{Z}$ and set $\epsilon:=\delta \circ \phi$. Then there is a morphism $\pi_{S}: \mathcal{X} \rightarrow \mathbb{P}:=\mathbb{P}\left(\epsilon_{*}\left(K_{\mathcal{Y} / S}^{\vee}\right)\right)$, which defines a family of elliptic fibrations. Choosing a sufficiently general family, we may assume that $\left.\pi_{S}\right|_{\xi}: \mathcal{X}_{\xi} \rightarrow \mathbb{P}_{k(\xi)}^{1}$ is an elliptic surface such that every fibre is irreducible. Let $\mathcal{O}_{\mathcal{X}}(1)$ be a relative ample line bundle on $\mathcal{X}$ which is sufficiently close to the pull-back of an ample line bundle on $\mathbb{P}$. For a line bundle $\mathcal{L}$ on $\mathcal{X}$ such that $c_{1}\left(\mathcal{L}_{s_{0}}\right)=c_{1}$, we shall consider the relative moduli space $\mathcal{M}(r, \mathcal{L}, \Delta) \rightarrow S$ of stable sheaves $E$ of rank $r$ on $\mathcal{X}_{s}, s \in S$ such that $c_{1}(E)=\mathcal{L}_{s}$ and $\Delta(E)=\Delta$. By Maruyama [Ma1, Cor. 5.9.1, Prop. 6.7], $\mathcal{M}(r, \mathcal{L}, \Delta)$ is smooth and projective over $S$. By Theorem 1.14, the generic fibre is irreducible, and hence every fibre is irreducible. Thus $M(\Delta)$ is irreducible. Since $M(\Delta)$ contain an irreducible component which is birational to $S^{n} X$ for some $n$ (see the proof of Theorem 1.14), $M(\Delta)$ is a rational variety.

Lemma 2.3. Let $\phi: \widetilde{X} \rightarrow X$ be a one point blow-up of a surface $X$ and $E$ a simple torsion free sheaf of rank $r$ on $X$ which is locally free at the center of the blow-up. Let $C_{1}$ be the exceptional divisor of $\phi$ and $\phi^{*} E \rightarrow \mathcal{O}_{C_{1}}^{\oplus k}$, $0<k<r$ a surjective homomorphism. We set $E^{\prime}:=\operatorname{ker}\left(\phi^{*} E \rightarrow \mathcal{O}_{C_{1}}^{\oplus k}\right)$. Then $E^{\prime}$ is also a simple torsion free sheaf.

Proof. We note that $\operatorname{Ext}^{1}\left(\mathcal{O}_{C_{1}}^{\oplus k}, E\right) \cong H^{1}\left(C_{1}, E^{\vee} \otimes \mathcal{O}_{C_{1}}\left(K_{\widetilde{X}}\right)^{\oplus k}\right) \cong$ $H^{1}\left(C_{1}, \mathcal{O}_{C_{1}}(-1)^{\oplus r k}\right)=0$. By the exact sequence $0 \rightarrow E^{\prime} \rightarrow E \rightarrow \mathcal{O}_{C_{1}}^{\oplus k} \rightarrow 0$, we see that $\operatorname{Hom}(E, E) \cong \operatorname{Hom}\left(E^{\prime}, E\right)$. Since $\operatorname{Hom}\left(E^{\prime}, E^{\prime}\right) \rightarrow \operatorname{Hom}\left(E^{\prime}, E\right)$ is injective, we get that $\operatorname{Hom}\left(E^{\prime}, E^{\prime}\right)=\mathbb{C}$.

COROLlary 2.4. Let $E$ be a simple torsion free sheaf of rank $r$ on $X$ with $c_{1}(E)=c_{1}$ and $\Delta(E)=(\Delta)$ which is locally free at the center 
of a blow-up $\phi: \widetilde{X} \rightarrow X$, and $E^{\prime}$ the kernel of a surjective homomorphism $\phi^{*} E \rightarrow \mathcal{O}_{C_{1}}^{\oplus k}, 0 \leq k<r$. We set $\Delta\left(E^{\prime}\right)=\Delta^{\prime}$. Then, if $\operatorname{Spl}\left(r, \phi^{*} c_{1}-k C_{1}, \Delta^{\prime}\right)$ is irreducible, $\operatorname{Spl}\left(r, c_{1}, \Delta\right)$ is also irreducible.

Proof. Let $\operatorname{Spl}\left(r, \phi^{*} c_{1}, \Delta\right)^{0}$ be the open subscheme of $\operatorname{Spl}\left(r, \phi^{*} c_{1}, \Delta\right)$ of elements $E$ such that $\left.E\right|_{C_{1}} \cong \mathcal{O}_{C_{1}}^{\oplus r}$. Then $\phi^{*}: \operatorname{Spl}\left(r, c_{1}, \Delta\right)^{\prime} \rightarrow \operatorname{Spl}\left(r, \phi^{*} c_{1}, \Delta\right)^{0}$ is an isomorphism, where $\operatorname{Spl}\left(r, c_{1}, \Delta\right)^{\prime}$ is the open dense subspace of $\operatorname{Spl}\left(r, c_{1}, \Delta\right)$ consisting of $E$ such that $E$ is locally free at the center of the blow-up. For an $E \in \operatorname{Spl}\left(r, \phi^{*} c_{1}, \Delta\right)^{0}$, the quotients $\phi^{*} E \rightarrow \mathcal{O}_{C_{1}}^{\oplus k}$ is parametrized by the Grassmannian variety $G\left(H^{0}\left(C_{1},\left.E\right|_{C_{1}} ^{\vee}\right), k\right)$. Let $\operatorname{Spl}\left(r, \phi^{*} c_{1}-\right.$ $\left.k C_{1}, \Delta^{\prime}\right)^{0}$ be the open subscheme of $\operatorname{Spl}\left(r, \phi^{*} c_{1}-k C_{1}, \Delta^{\prime}\right)$ of elements $E^{\prime}$ such that $\left.E^{\prime}\right|_{C_{1}} \cong \mathcal{O}_{C_{1}}(1)^{\oplus k} \oplus \mathcal{O}_{C_{1}}^{\oplus(r-k)}$. By using Lemma 2.3, we can show that there is an open subscheme $U$ of $\operatorname{Spl}\left(r, \phi^{*} c_{1}-k C_{1}, \Delta^{\prime}\right)^{0}$ and a surjective morphism $U \rightarrow \operatorname{Spl}\left(r, \phi^{*} c_{1}, \Delta\right)^{0}$ such that every fibre is a Grassmannian variety. Hence, the irreducibility of $\operatorname{Spl}\left(r, \phi^{*} c_{1}-k C_{1}, \Delta^{\prime}\right)$ implies that of $\operatorname{Spl}\left(r, c_{1}, \Delta\right)$.

Proposition 2.5. Let $X$ be a Del Pezzo surface and $c_{1}$ an element of $\mathrm{NS}(X)$. Then $\operatorname{Spl}\left(r, c_{1}, \Delta\right)$ is irreducible.

Proof. Let $X$ be a Del Pezzo surface which is an $n$-points blow-ups of $\mathbb{P}^{2}$. If $n<8$, then we shall take a blow-ups $X^{\prime}$ of $X$ at general $8-n$ points. Then $\left|K_{X^{\prime}}^{\vee}\right|$ is a linear pencil with a base point. Let $X^{\prime \prime}$ be the blow-up of $X^{\prime}$ at the base point. Then $X^{\prime \prime}$ is an elliptic surface with a section. By using Corollary 2.4 and Proposition 2.2, we get our claim.

\section{$\S 3$. Moduli spaces on Abelian surfaces}

\section{1.}

For a manifold $V$ and $\alpha \in H^{*}(V, \mathbb{Z}),[\alpha]_{i} \in H^{i}(V, \mathbb{Z})$ denotes the $i$-th component of $\alpha$. Let $K(V)$ be the Grothendieck group of $V$. Let $p: X \rightarrow$ $\operatorname{Spec}(\mathbb{C})$ be an Abelian surface over $\mathbb{C}$. We set

$$
\left\{\begin{array}{l}
H^{e v}(X, \mathbb{Z}):=H^{0}(X, \mathbb{Z}) \oplus H^{2}(X, \mathbb{Z}) \oplus H^{4}(X, \mathbb{Z}) \\
H^{\text {odd }}(X, \mathbb{Z}):=H^{1}(X, \mathbb{Z}) \oplus H^{3}(X, \mathbb{Z}) .
\end{array}\right.
$$

Let $E_{0}$ be an element of $M_{H}\left(r, c_{1}, \Delta\right)$. We set

$$
H\left(r, c_{1}, \Delta\right):=\left\{\alpha \in H^{e v}(X, \mathbb{Z}) \mid\left[p_{*}\left(\left(\operatorname{ch} E_{0}\right) \alpha\right)\right]_{0}=0\right\}
$$


Let $\mathcal{F}$ be a quasi-universal family of similitude $\rho$ on $M_{H}\left(r, c_{1}, \Delta\right) \times X[\mathrm{Mu} 3$, Thm. A.5]. Then Mukai [Mu3], [Mu5] and Drezet [D], [D-N] defines a homomorphism

$$
\kappa_{2}: H\left(r, c_{1}, \Delta\right) \longrightarrow H^{2}\left(M_{H}\left(r, c_{1}, \Delta\right), \mathbb{Z}\right)
$$

such that

$$
\kappa_{2}(\alpha)=\frac{1}{\rho}\left[p_{M_{H}\left(r, c_{1}, \Delta\right) *}(\operatorname{ch}(\mathcal{F}) \alpha)\right]_{2} .
$$

Remark 3.1. In the notation of Mukai [Mu5, Sect. 5], $\kappa_{2}(\alpha)=-\theta_{v}\left(\alpha^{\vee}\right)$ and $H\left(r, c_{1}, \Delta\right)=v^{\perp}$, where $v:=\left(r, c_{1},\left(c_{1}^{2}\right) / 2 r-\Delta\right) \in H^{e v}(X, \mathbb{Z})$ is the Chern character of $E_{0}$ and ${ }^{\vee}: H^{e v}(X, \mathbb{Z}) \rightarrow H^{e v}(X, \mathbb{Z})$ is the automorphism sending $\alpha=\alpha_{0}+\alpha_{2}+\alpha_{4}, \alpha_{i} \in H^{2 i}(X, \mathbb{Z})$ to $\alpha^{\vee}=\alpha_{0}-\alpha_{2}+\alpha_{4}$. Since we used Drezet's notation in [Y2], [Y3], we shall use Drezet's homomorphism in this note.

We also consider the homomorphism:

$$
\kappa_{1}: H^{\text {odd }}(X, \mathbb{Z}) \longrightarrow H^{1}\left(M_{H}\left(r, c_{1}, \Delta\right), \mathbb{Z}\right)
$$

such that

$$
\kappa_{1}(\alpha)=\frac{1}{\rho}\left[p_{M_{H}\left(r, c_{1}, \Delta\right) *}(\operatorname{ch}(\mathcal{F}) \alpha)\right]_{1} .
$$

We note that $\kappa_{1}$ and $\kappa_{2}$ do not depend on the choice of $\mathcal{F}$. In this section, we shall prove the following theorem.

TheOrem 3.1. Let $c_{1}$ be an element of $\mathrm{NS}(X)$ such that $c_{1} \bmod$ $r H^{2}(X, \mathbb{Z})$ is a primitive element of $H^{2}(X, \mathbb{Z} / r \mathbb{Z})$ and $H$ a general ample divisor. We assume that $\operatorname{dim} M_{H}\left(r, c_{1}, \Delta\right)=2 r \Delta+2 \geq 6$. Let $\mathfrak{a}$ : $M_{H}\left(r, c_{1}, \Delta\right) \rightarrow \operatorname{Alb}\left(M_{H}\left(r, c_{1}, \Delta\right)\right)$ be an Albanese map. Then the following holds.

(1) $\kappa_{1}$ is an isomorphism and $\kappa_{2}$ is injective.

(2)

$$
\begin{aligned}
& H^{2}\left(M_{H}\left(r, c_{1}, \Delta\right), \mathbb{Z}\right) \\
& \quad=\kappa_{2}\left(H\left(r, c_{1}, \Delta\right)\right) \oplus \mathfrak{a}^{*} H^{2}\left(\operatorname{Alb}\left(M_{H}\left(r, c_{1}, \Delta\right), \mathbb{Z}\right)\right. \\
& \quad=\kappa_{2}\left(H\left(r, c_{1}, \Delta\right)\right) \oplus \bigwedge^{2} \kappa_{1}\left(H^{\text {odd }}(X, \mathbb{Z})\right) .
\end{aligned}
$$

(3.8) $\operatorname{NS}\left(M_{H}\left(r, c_{1}, \Delta\right)\right)=\kappa_{2}\left(H\left(r, c_{1}, \Delta\right)_{a l g}\right) \oplus \mathfrak{a}^{*} \operatorname{NS}\left(\operatorname{Alb}\left(M_{H}\left(r, c_{1}, \Delta\right)\right)\right.$, where $H\left(r, c_{1}, \Delta\right)_{\text {alg }}:=\left(H^{0}(X, \mathbb{Z}) \oplus \mathrm{NS}(X) \oplus H^{4}(X, \mathbb{Z})\right) \cap H\left(r, c_{1}, \Delta\right)$. 


\section{2.}

We first assume that $X$ is a product of elliptic curves. Let $C_{1}$ and $C_{2}$ be elliptic curves and set $X=C_{1} \times C_{2}$. Since we use products such as $X \times X \times \cdots \times X$ and $C_{1} \times C_{1} \times \cdots \times C_{1}$, for convenience sake, we shall introduce indices of $C_{1}, C_{2}$ and $X$. We set $C_{k}^{i}:=C_{k}$ and $X^{i}:=C_{1}^{i} \times C_{2}^{i}$ for $i=-1,0,1, \ldots, n(\geq 2)$, and $k=1,2$. We set $a:=-1$. We shall construct a family of stable sheaves on $X^{a}(=X)$. Let $\Delta_{k}^{i, j}$ be the diagonal of $C_{k}^{i} \times C_{k}^{j}=C_{k} \times C_{k}$. Let $p_{k}^{i}$ be a point of $C_{k}^{i}$. We also denote $c_{1}\left(\mathcal{O}\left(p_{k}^{i}\right)\right)$ by $p_{k}^{i}$. For simplicity, we denote the pull-backs of $p_{k}^{i}$ and $\Delta_{k}^{i, j}$ to $X^{0} \times\left(X^{1} \times\right.$ $\left.X^{2} \times \cdots \times X^{n}\right) \times X^{a}$ by $p_{k}^{i}$ and $\Delta_{k}^{i, j}$ respectively. Let $\Delta_{X}^{i, j, k}$ be the pullback of the diagonal of $X^{i} \times X^{j} \times X^{k}$ to $X^{1} \times X^{2} \times \cdots \times X^{n}$ and $\Delta_{X}^{i, j}$ that of $X^{i} \times X^{j}$ to $X^{1} \times X^{2} \times \cdots \times X^{n}$. We set $Z:=\bigcup_{i<j<k} \Delta_{X}^{i, j, k}$. Let $\phi: Y \rightarrow\left(X^{1} \times X^{2} \times \cdots \times X^{n}\right) \backslash Z$ be the blow-up of $\left(X^{1} \times X^{2} \times \cdots \times X^{n}\right) \backslash Z$ at the subscheme $\bigcup_{i<j} \Delta_{X}^{i, j} \backslash Z$. We set $E^{i, j}:=\phi^{-1}\left(\Delta_{X}^{i, j} \backslash Z\right)$. For $\alpha \in H^{*}(X, \mathbb{Z})$ and the projection $\varpi_{i}: X^{0} \times Y \times X^{a} \rightarrow X^{i}=X, i=0,1, \ldots, n, a$, we denote the pull-back of $\alpha$ to $X^{0} \times Y \times X^{a}$ by $\alpha^{i}$. Then $H^{2}\left(\operatorname{Hilb}_{X}^{n}, \mathbb{Z}\right) \cong H^{2}(Y, \mathbb{Z})^{\mathfrak{S}_{n}}$ and $H^{2}(Y, \mathbb{Z})^{\mathfrak{S}_{n}}$ is generated by $\sum_{i=1}^{n} e^{i}, \sum_{i<j}\left(f^{i} \cdot g^{j}-g^{i} \cdot f^{j}\right)$ and $\sum_{i<j} E^{i, j}$ where $e \in H^{2}(X, \mathbb{Z})$ and $f, g \in H^{1}(X, \mathbb{Z})$. Let $\mathfrak{a}: X^{0} \times \operatorname{Hilb}_{X}^{n} \rightarrow X^{0} \times X$ be the Albanese map such that $\mathfrak{a}\left(\left(x, I_{Z}\right)\right)=\left(x, \sum_{i=1}^{n} x_{i}\right)$ for reduced subscheme $Z=\bigcup_{i}\left\{x_{i}\right\}$.

LEMMA 3.2. (1) Let $F$ be a vector bundle on $C_{2}^{0} \times C_{2}^{a}$ such that $\left.F\right|_{\{t\} \times C_{2}^{a}, t \in C_{2}^{0}}$ is a stable vector bundle of rank $r$ on $C_{2}^{a}$ with $\left.\operatorname{det} F\right|_{\{t\} \times C_{2}^{a}} \cong$ $\left.\mathcal{O}\left(\Delta_{2}^{0, a}+(d-1) p_{2}^{a}\right)\right|_{\{t\} \times C_{2}^{a} \text {. Then, }}$

$$
\left\{\begin{array}{l}
c_{1}(F)=\Delta_{2}^{0, a}+(d-1) p_{2}^{a}+\left(r_{1}-1+k r\right) p_{2}^{0}, \quad k \in \mathbb{Z}, \\
\operatorname{ch}_{2}(F)=\frac{1}{2 r}\left(c_{1}(F)^{2}\right) .
\end{array}\right.
$$

If $k=0$, then $\operatorname{ch}_{2}(F)=d_{1} p_{2}^{0} \cdot p_{2}^{a}$.

(2) Let $F_{i}(1 \leq i \leq n)$ be a vector bundle on $C_{2}^{i} \times C_{2}^{a}$ such that $\left.F_{i}\right|_{\{t\} \times C_{2}^{a}}$, $t \in C_{2}^{i}$ is a stable vector bundle of rank $r_{2}$ on $C_{2}^{a}$ with $\left.\operatorname{det} F_{i}\right|_{\{t\} \times C_{2}^{a}} \cong$ $\left.\mathcal{O}\left(\Delta_{2}^{i, a}+\left(d_{2}-1\right) p_{2}^{a}\right)\right|_{\{t\} \times C_{2}^{a} \text {. Then, }}$

$$
\left\{\begin{array}{l}
c_{1}\left(F_{i}\right)=\Delta_{2}^{i, a}+\left(d_{2}-1\right) p_{2}^{a}+\left(r_{1}-1+k r_{2}\right) p_{2}^{i}, \quad k \in \mathbb{Z}, \\
\operatorname{ch}_{2}\left(F_{i}\right)=\frac{1}{2 r_{2}}\left(c_{1}\left(F_{i}\right)^{2}\right) .
\end{array}\right.
$$

If $k=0$, then $\operatorname{ch}_{2}\left(F_{i}\right)=d_{1} p_{2}^{i} \cdot p_{2}^{a}$. 
Proof. We shall only prove (1). We set $c_{1}(F)=\Delta_{2}^{0, a}+(d-1) p_{2}^{a}+\left(r_{1}-\right.$

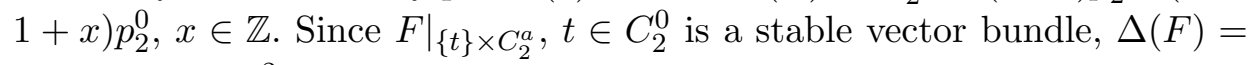
$c_{2}(F)-\left(c_{1}(F)^{2}\right)(r-1) / 2 r=0$. Hence we get that $\operatorname{ch}_{2}(F)=-\left(c_{2}(F)-\right.$ $\left.\left(c_{1}(F)^{2}\right) / 2\right)=\left(c_{1}(F)^{2}\right) / 2 r$. We note that $c_{2}(F)=\left(d\left(r_{1}+x\right)-1\right)(r-1) / r$ is an integer. Hence $d\left(r_{1}+x\right)-1=r d_{1}+d x$ is a multiple of $r$. Since $(r, d)=1$, $x$ is a multiple of $r$. We also see that $\left(c_{1}(F)^{2}\right) / 2 r=d_{1} p_{2}^{0} \cdot p_{2}^{a}$ for the case $x=0$.

Let $F$ and $F_{i}$ be vector bundles in Lemma 3.2 and assume that $k=0$. We also denote the pull-backs of $F$ and $F_{i}$ to $C_{2}^{0} \times C_{2}^{i} \times C_{2}^{a}$ by $F$ and $F_{i}$ respectively. Let $q_{C_{2}^{0} \times C_{2}^{i}}: C_{2}^{0} \times C_{2}^{i} \times C_{2}^{a} \rightarrow C_{2}^{0} \times C_{2}^{i}$ be the projection. We set $\mathcal{L}:=\operatorname{Hom}_{q_{C_{2}^{0} \times C_{2}^{a}}}\left(F, F_{i}\right)$. Then $c_{1}(\mathcal{L})=-\Delta_{2}^{0, i}$.

Proof. By using the Grothendieck-Riemann-Roch theorem and the above lemma, we see that

$$
\begin{aligned}
c_{1}(\mathcal{L}) & =\left[q_{C_{2}^{0} \times C_{2}^{i} *}\left(\operatorname{ch}\left(F^{\vee}\right) \operatorname{ch}\left(F_{i}\right)\right)\right]_{2} \\
& =\left[q_{C_{2}^{0} \times C_{2}^{i} *}\left(r-c_{1}(F)+\frac{1}{2 r}\left(c_{1}(F)^{2}\right)\right)\left(r_{2}+c_{1}\left(F_{i}\right)+\frac{1}{2 r_{2}}\left(c_{1}\left(F_{i}\right)^{2}\right)\right)\right]_{2} \\
& =\left[q_{C_{2}^{0} \times C_{2}^{i} *}\left(r r_{2}+\left(r c_{1}\left(F_{i}\right)-r_{2} c_{1}(F)\right)+\frac{1}{2 r r_{2}}\left(\left(r c_{1}\left(F_{i}\right)-r_{2} c_{1}(F)\right)^{2}\right)\right)\right]_{2} \\
& =\frac{1}{2 r r_{2}}\left[q_{C_{2}^{0} \times C_{2}^{i} *}\left(\left(r c_{1}\left(F_{i}\right)-r_{2} c_{1}(F)\right)^{2}\right)\right]_{2} \\
& =-\Delta_{2}^{0, i} .
\end{aligned}
$$

Let $Y_{0}$ be the complement of the closed subset $W:=\bigcup_{i<j<k}\left(\widetilde{\Delta}_{1}^{i, j} \cap\right.$ $\left.\widetilde{\Delta}_{1}^{j, k}\right) \cup \bigcup_{i<j}\left(\widetilde{\Delta}_{1}^{i, j} \cap E^{i, j}\right)$ of $Y$, where $\Delta_{1}^{i, j}=\widetilde{\Delta}_{1}^{i, j} \cup E^{i, j}$. Since codim $W=2$, $H^{2}\left(X^{0} \times Y_{0}, \mathbb{Z}\right) \cong H^{2}\left(X^{0} \times Y, \mathbb{Z}\right)$.

We shall construct a family of stable sheaves on $X$ parametrized by $X^{0} \times Y_{0}$. For simplicity, we denote the pull-backs of $F$ and $F_{i}$ to $X^{0} \times Y_{0} \times X^{a}$ by $F$ and $F_{i}$ respectively. Then there is a homomorphism:

$$
\Lambda: F \otimes \mathcal{O}\left(\Delta_{1}^{0, a}-p_{1}^{a}\right) \longrightarrow \bigoplus_{i=1}^{n}\left(\left.F_{i}\right|_{\Delta_{1}^{i, a}} \otimes L^{i}\right)
$$

where $L^{i}$ is a line bundle on $X^{0} \times Y_{0} \times X^{a}$ such that $c_{1}\left(L^{i}\right)=\Delta_{1}^{0, i}-$ $p_{1}^{i}+\Delta_{2}^{0, i}$. Let $\mathcal{E}$ be the kernel of this homomorphism and $\mathcal{Q}$ the cokernel. Then $\mathcal{Q} \cong \bigoplus_{i<j}\left(\left.\left(F_{i} / G_{j}\right)\right|_{\Delta_{1}^{i, a} \cap \widetilde{\Delta}_{1}^{i, j}} \otimes L^{i} \oplus\left(\left.F_{i} \otimes L^{i}\right|_{\Delta_{1}^{i, a}} \otimes \mathcal{O}_{E^{i, j}}\right)\right)$, where 
$G_{i}:=\operatorname{ker}\left(F \rightarrow F_{i}\right)$. We first assume that $r_{1} \leq r_{2}$. Then $\left.\left.G_{j}\right|_{\Delta_{1}^{i, a}} \rightarrow F_{i}\right|_{\Delta_{1}^{i, a}}$ is injective and $\left.\left(F_{i} / G_{j}\right)\right|_{\Delta_{1}^{i, a}}$ is flat over $X^{0} \times Y_{0}$. Hence we see that

$$
\operatorname{Tor}_{2} \mathcal{O}_{X^{0} \times Y_{0}}\left(\left.\left(F_{i} / G_{j}\right)\right|_{\Delta_{1}^{i, a} \cap \widetilde{\Delta}_{1}^{i, j}}, k(x)\right)=0, \quad x \in X^{0} \times Y_{0}
$$

Since $\left.F_{i} \otimes \mathcal{O}\left(\Delta_{2}^{0, i}\right)\right|_{\Delta_{1}^{i, a}}$ is also flat over $X^{0} \times Y_{0}$, we get that

$$
\operatorname{Tor}_{2} \mathcal{O}_{X^{0} \times Y_{0}}\left(\left.F_{i} \otimes \mathcal{O}\left(\Delta_{2}^{0, i}\right)\right|_{\Delta_{1}^{i, a}} \otimes \mathcal{O}_{E^{i, j}}, k(x)\right)=0, \quad x \in X^{0} \times Y_{0}
$$

Hence we see that $\operatorname{Tor}_{1} \mathcal{O}_{X^{0} \times Y_{0}}(\operatorname{im}(\Lambda), k(x))=0$, which implies that $\mathcal{E}$ is flat over $X^{0} \times Y_{0}$ and $\mathcal{E} \otimes k(x)$ is torsion free. Then $\mathcal{E}$ defines a family of stable sheaves on $X$ parametrized by $X^{0} \times Y_{0}$. It defines a morphism $X^{0} \times Y_{0} \rightarrow M\left(r, c_{1}, \Delta\right)$, which is $\mathfrak{S}_{n}$-invariant. Hence we get a morphism $\nu: X^{0} \times\left(Y_{0} / \mathfrak{S}_{n}\right) \rightarrow M\left(r, c_{1}, \Delta\right)$.

Let $\overline{\kappa_{2}}: H\left(r, c_{1}, \Delta\right) \rightarrow H^{2}\left(X^{0} \times Y_{0}, \mathbb{Z}\right) / \mathfrak{a}^{*} H^{2}\left(\operatorname{Alb}\left(X^{0} \times \operatorname{Hilb}_{X}^{n}\right), \mathbb{Z}\right)$ be the homomorphism sending $\alpha \in H\left(r, c_{1}, \Delta\right)$ to $\left[p_{X^{0} \times Y_{0} *}(\operatorname{ch}(\mathcal{E}) \alpha)\right]_{2} \bmod$ $\mathfrak{a}^{*} H^{2}\left(\operatorname{Alb}\left(X^{0} \times \operatorname{Hilb}_{X}^{n}\right), \mathbb{Z}\right)$. Since $\kappa_{2}$ does not depend on the choice of quasiuniversal families, we shall compute the image of $\overline{\kappa_{2}}$.

$$
\begin{aligned}
\operatorname{ch}(\mathcal{E})=\operatorname{ch} & \left.F \otimes \mathcal{O}\left(\Delta_{1}^{0, a}-p_{1}^{a}\right)\right)-\sum_{i=1}^{n} \operatorname{ch}\left(\left.F_{i} \otimes L^{i}\right|_{\Delta_{1}^{i, a}}\right) \\
& +\sum_{i<j} \operatorname{ch}\left(\left.F_{i} \otimes L^{i}\right|_{\Delta_{1}^{i, a}} \otimes \mathcal{O}_{E^{i, j}}\right)+\sum_{i<j} \operatorname{ch}\left(F_{i} /\left.G_{j} \otimes L^{i}\right|_{\Delta_{1}^{i, a}} \otimes \mathcal{O}_{\widetilde{\Delta}_{1}^{i, j}}\right) \\
=(r & \left.+c_{1}(F)+d_{1} p_{2}^{0} \cdot p_{2}^{a}\right)\left(1+\Delta_{1}^{0, a}-p_{1}^{a}-p_{1}^{0} \cdot p_{1}^{a}\right) \\
& -\sum_{i=1}^{n} \Delta_{1}^{i, a}\left(r_{2}+c_{1}\left(F_{i}\right)+d_{1} p_{2}^{i} \cdot p_{2}^{a}\right)\left(\operatorname{ch} L^{i}\right) \\
& +\sum_{i<j} \operatorname{ch}\left(\left.F_{i} \otimes L^{i}\right|_{\Delta_{1}^{i, a}} \otimes \mathcal{O}_{E^{i, j}}\right)+\sum_{i<j} \operatorname{ch}\left(F_{i} /\left.G_{j} \otimes L^{i}\right|_{\Delta_{1}^{i, a}} \otimes \mathcal{O}_{\widetilde{\Delta}_{1}^{i, j}}\right) .
\end{aligned}
$$

Since $\left[p_{X^{0} \times Y_{0} *}\left(\operatorname{ch}\left(F \otimes \mathcal{O}\left(\Delta_{1}^{0, a}-p_{1}^{a}\right)\right) \alpha^{a}\right)\right]_{2} \equiv 0, \sum_{i=1}^{n} \Delta_{1}^{0, i}-p_{1}^{i} \equiv 0 \bmod$ $\mathfrak{a}^{*} H^{2}\left(\operatorname{Alb}\left(X^{0} \times \operatorname{Hilb}_{X}^{n}\right), \mathbb{Z}\right)$, we get that

$$
\begin{aligned}
\overline{\kappa_{2}}(\alpha)=- & \sum_{i=1}^{n}\left[p_{X^{0} \times Y_{0} *}\left(\Delta_{1}^{i, a}\left(r_{2}+c_{1}\left(F_{i}\right)+d_{1} p_{2}^{i} \cdot p_{2}^{a}\right)\left(1+\Delta_{2}^{0, i}\right) \alpha^{a}\right)\right]_{2} \\
& +\sum_{i<j}\left[p_{X^{0} \times Y_{0} *}\left(\operatorname{ch}\left(\left.F_{i} \otimes \mathcal{O}\left(\Delta_{2}^{0, i}\right)\right|_{\Delta_{1}^{i, a}} \otimes \mathcal{O}_{E^{i, j}}\right) \alpha^{a}\right)\right]_{2}
\end{aligned}
$$




$$
+\sum_{i<j}\left[p_{X^{0} \times Y_{0} *}\left(\operatorname{ch}\left(F_{i} /\left.G_{j} \otimes \mathcal{O}\left(\Delta_{2}^{0, i}\right)\right|_{\Delta_{1}^{i, a}} \otimes \mathcal{O}_{\widetilde{\Delta}_{1}^{i, j}}\right) \alpha^{a}\right)\right]_{2} .
$$

Let $\alpha=x_{1}+x_{2} p_{1}+x_{3} p_{2}+x_{4} p_{1} \cdot p_{2}+D$ be an element of $H\left(r, c_{1}, \Delta\right)$, $D \in H^{1}\left(C_{1}, \mathbb{Z}\right) \otimes H^{1}\left(C_{2}, \mathbb{Z}\right)$. Then we see that $0=\left[p_{*}\left(\left(\operatorname{ch} E_{0}\right) \alpha\right)\right]_{0}=\left[p_{*}((r+\right.$ $\left.\left.\left.d p_{2}-r_{2} n p_{1}-d_{2} n p_{1} \cdot p_{2}\right) \alpha\right)\right]_{0}=-d_{2} n x_{1}-r_{2} n x_{3}+d x_{2}+r x_{4}$. Thus $\alpha$ satisfies

$$
d x_{2}+r x_{4}=d_{2} n x_{1}+r_{2} n x_{3} .
$$

By a simple calculation, we get that

$$
\begin{aligned}
& \left\{\begin{array}{l}
{\left[p_{X^{0} \times Y_{0^{*}}}\left(\operatorname{ch}\left(\left.F_{i} \otimes \mathcal{O}\left(\Delta_{2}^{0, i}\right)\right|_{\Delta_{1}^{i, a}}\right)\right)\right]_{2}=d_{2} \Delta_{2}^{0, i}+d_{1} p_{2}^{i}} \\
{\left[p_{X^{0} \times Y_{0^{*}}}\left(\operatorname{ch}\left(\left.F_{i} \otimes \mathcal{O}\left(\Delta_{2}^{0, i}\right)\right|_{\Delta_{1}^{i, a}}\right) p_{2}^{a}\right)\right]_{2}=r_{2} \Delta_{2}^{0, i}+r_{1} p_{2}^{i}} \\
{\left[p_{X^{0} \times Y_{0} *}\left(\operatorname{ch}\left(\left.F_{i} \otimes \mathcal{O}\left(\Delta_{2}^{0, i}\right)\right|_{\Delta_{1}^{i, a}}\right) p_{1}^{a}\right)\right]_{2}=d_{2} p_{1}^{i}} \\
{\left[p_{X^{0} \times Y_{0^{*}}}\left(\operatorname{ch}\left(\left.F_{i} \otimes \mathcal{O}\left(\Delta_{2}^{0, i}\right)\right|_{\Delta_{1}^{i, a}}\right) D^{a}\right)\right]_{2}=D^{i}} \\
{\left[p_{X^{0} \times Y_{0^{*}}}\left(\operatorname{ch}\left(\left.F_{i} \otimes \mathcal{O}\left(\Delta_{2}^{0, i}\right)\right|_{\Delta_{1}^{i, a}}\right)\left(p_{1}^{a} \cdot p_{2}^{a}\right)\right)\right]_{2}=r_{2} p_{1}^{i},}
\end{array}\right. \\
& \left\{\begin{array}{l}
{\left[p_{X^{0} \times Y_{0^{*}}}\left(\operatorname{ch}\left(F_{i} /\left.G_{j} \otimes \mathcal{O}\left(\Delta_{2}^{0, i}\right)\right|_{\Delta_{1}^{i, a}} \otimes \mathcal{O}_{\widetilde{\Delta}_{1}^{i, j}}\right)\right)\right]_{2}=\left(2 d_{2}-d\right) \widetilde{\Delta}_{1}^{i, j}} \\
{\left[p_{X^{0} \times Y_{0} *}\left(\operatorname{ch}\left(F_{i} /\left.G_{j} \otimes \mathcal{O}\left(\Delta_{2}^{0, i}\right)\right|_{\Delta_{1}^{i, a}} \otimes \mathcal{O}_{\widetilde{\Delta}_{1}^{i, j}}\right) p_{2}^{a}\right)\right]_{2}=\left(2 r_{2}-r\right) \widetilde{\Delta}_{1}^{i, j}} \\
{\left[p_{X^{0} \times Y_{0^{*}}}\left(\operatorname{ch}\left(F_{i} /\left.G_{j} \otimes \mathcal{O}\left(\Delta_{2}^{0, i}\right)\right|_{\Delta_{1}^{i, a}} \otimes \mathcal{O}_{\widetilde{\Delta}_{1}^{i, j}}\right) p_{1}^{a}\right)\right]_{2}=0} \\
{\left[p_{X^{0} \times Y_{0} *}\left(\operatorname{ch}\left(F_{i} /\left.G_{j} \otimes \mathcal{O}\left(\Delta_{2}^{0, i}\right)\right|_{\Delta_{1}^{i, a}} \otimes \mathcal{O}_{\widetilde{\Delta}_{1}^{i, j}}\right) D^{a}\right)\right]_{2}=0} \\
{\left[p_{X^{0} \times Y_{0} *}\left(\operatorname{ch}\left(F_{i} /\left.G_{j} \otimes \mathcal{O}\left(\Delta_{2}^{0, i}\right)\right|_{\Delta_{1}^{i, a}} \otimes \mathcal{O}_{\widetilde{\Delta}_{1}^{i, j}}\right)\left(p_{1}^{a} \cdot p_{2}^{a}\right)\right)\right]_{2}=0,}
\end{array}\right.
\end{aligned}
$$

and

$$
\left\{\begin{array}{l}
{\left[p_{X^{0} \times Y_{0} *}\left(\operatorname{ch}\left(\left.F_{i} \otimes \mathcal{O}\left(\Delta_{2}^{0, i}\right)\right|_{\Delta_{1}^{i, a}} \otimes \mathcal{O}_{E^{i, j}}\right)\right)\right]_{2}=d_{2} E^{i, j}} \\
{\left[p_{X^{0} \times Y_{0^{*}}}\left(\operatorname{ch}\left(\left.F_{i} \otimes \mathcal{O}\left(\Delta_{2}^{0, i}\right)\right|_{\Delta_{1}^{i, a}} \otimes \mathcal{O}_{E^{i, j}}\right) p_{2}^{a}\right)\right]_{2}=r_{2} E^{i, j}} \\
{\left[p_{X^{0} \times Y_{0^{*}}}\left(\operatorname{ch}\left(\left.F_{i} \otimes \mathcal{O}\left(\Delta_{2}^{0, i}\right)\right|_{\Delta_{1}^{i, a}} \otimes \mathcal{O}_{E^{i, j}}\right) p_{1}^{a}\right)\right]_{2}=0} \\
{\left[p_{X^{0} \times Y_{0} *}\left(\operatorname{ch}\left(\left.F_{i} \otimes \mathcal{O}\left(\Delta_{2}^{0, i}\right)\right|_{\Delta_{1}^{i, a}} \otimes \mathcal{O}_{E^{i, j}}\right) D^{a}\right)\right]_{2}=0} \\
{\left[p_{X^{0} \times Y_{0} *}\left(\operatorname{ch}\left(\left.F_{i} \otimes \mathcal{O}\left(\Delta_{2}^{0, i}\right)\right|_{\Delta_{1}^{i, a}} \otimes \mathcal{O}_{E^{i, j}}\right)\left(p_{1}^{a} \cdot p_{2}^{a}\right)\right)\right]_{2}=0}
\end{array}\right.
$$

where $D \in H^{1}\left(C_{1}, \mathbb{Z}\right) \otimes H^{1}\left(C_{2}, \mathbb{Z}\right)$. Hence we get that

$$
\begin{aligned}
\overline{\kappa_{2}}(\alpha)=- & \sum_{i=1}^{n}\left(d_{2} x_{1}+r_{2} x_{3}\right) \Delta_{2}^{0, i}-\sum_{i=1}^{n}\left(d_{2} x_{2}+r_{2} x_{4}\right) p_{1}^{i} \\
& -\sum_{i=1}^{n}\left(d_{1} x_{1}+r_{1} x_{3}\right) p_{2}^{i}-\sum_{i=1}^{n} D^{i} \\
& +\sum_{i<j}\left(\left(2 d_{2}-d\right) x_{1}+\left(2 r_{2}-r\right) x_{3}\right) \widetilde{\Delta}_{1}^{i, j}+\sum_{i<j}\left(d_{2} x_{1}+r_{2} x_{3}\right) E .
\end{aligned}
$$


We note that

$$
\left\{\begin{array}{l}
\sum_{i=1}^{n} \Delta_{2}^{0, i} \equiv \sum_{i=1}^{n} p_{2}^{i} \bmod \mathfrak{a}^{*} H^{2}\left(\operatorname{Alb}\left(X^{0} \times \operatorname{Hilb}_{X}^{n}\right), \mathbb{Z}\right) \\
\sum_{i<j} \Delta_{1}^{i, j} \equiv n \sum_{i=1}^{n} p_{1}^{i} \bmod \mathfrak{a}^{*} H^{2}\left(\operatorname{Alb}\left(X^{0} \times \operatorname{Hilb}_{X}^{n}\right), \mathbb{Z}\right) \\
\widetilde{\Delta}_{1}^{i, j}=\Delta_{1}^{i, j}-E^{i, j}
\end{array}\right.
$$

Therefore we get that

$$
\overline{\kappa_{2}}(\alpha)=y_{1}\left(\sum_{i=1}^{n} p_{2}^{i}\right)+y_{2}\left(\sum_{i=1}^{n} p_{1}^{i}\right)+y_{3}\left(\sum_{i<j} E^{i, j}\right)-\sum_{i=1}^{n} D^{i}
$$

where

$$
\left\{\begin{array}{l}
y_{1}=-\left(d x_{1}+r x_{3}\right) \\
y_{2}=-\left\{\left(d_{2} x_{2}+r_{2} x_{4}\right)-n\left(\left(2 d_{2}-d\right) x_{1}+\left(2 r_{2}-r\right) x_{3}\right)\right\} \\
y_{3}=\left(d_{1} x_{1}+r_{1} x_{3}\right) \\
y_{4}=d x_{2}+r x_{4}-n\left(d_{2} x_{1}+r_{2} x_{3}\right)
\end{array}\right.
$$

Since $d r_{1}-r d_{1}=d_{2} r-d r_{2}=1$, the homomorphism $\psi: \mathbb{Z}^{\oplus 4} \rightarrow \mathbb{Z}^{\oplus 4}$ sending $\left(x_{1}, x_{2}, x_{3}, x_{4}\right)$ to $\left(y_{1}, y_{2}, y_{3}, y_{4}\right)$ is an isomorphism. The condition (3.14) implies that $y_{4}=0$. Therefore,

$$
\overline{\kappa_{2}}: H\left(r, c_{1}, \Delta\right) \longrightarrow H^{2}\left(X^{0} \times Y_{0}, \mathbb{Z}\right)^{\mathfrak{S}_{n}} / \mathfrak{a}^{*} H^{2}\left(\operatorname{Alb}\left(X^{0} \times \operatorname{Hilb}_{X}^{n}\right), \mathbb{Z}\right)
$$

is an isomorphism. Since $H^{2}\left(X^{0} \times Y_{0}, \mathbb{Z}\right)^{\mathfrak{S}_{n}} \cong H^{2}\left(X^{0} \times \operatorname{Hilb}_{X}^{n}, \mathbb{Z}\right)$, we get that

$$
H\left(r, c_{1}, \Delta\right) \longrightarrow H^{2}\left(X^{0} \times \operatorname{Hilb}_{X}^{n}, \mathbb{Z}\right) / \mathfrak{a}^{*} H^{2}\left(\operatorname{Alb}\left(X^{0} \times \operatorname{Hilb}_{X}^{n}\right), \mathbb{Z}\right)
$$

is an isomorphism.

We next treat the case $r_{1}>r_{2}$. Since $G_{j} \rightarrow F_{i}$ is surjective, we get that

$$
\begin{aligned}
\overline{\kappa_{2}}(\alpha)=- & \sum_{i=1}^{n}\left(d_{2} x_{1}+r_{2} x_{3}\right) \Delta_{2}^{0, i}-\sum_{i=1}^{n}\left(d_{2} x_{2}+r_{2} x_{4}\right) p_{1}^{i} \\
& -\sum_{i=1}^{n}\left(d_{1} x_{1}+r_{1} x_{3}\right) p_{2}^{i}-\sum_{i=1}^{n} D^{i}+\sum_{i<j}\left(d_{2} x_{1}+r_{2} x_{3}\right) E .
\end{aligned}
$$


In the same way as in the case $r_{1} \leq r_{2}$, we see that

$$
H\left(r, c_{1}, \Delta\right) \longrightarrow H^{2}\left(X^{0} \times \operatorname{Hilb}_{X}^{n}, \mathbb{Z}\right) / \mathfrak{a}^{*} H^{2}\left(\operatorname{Alb}\left(X^{0} \times \operatorname{Hilb}_{X}^{n}\right), \mathbb{Z}\right)
$$

is an isomorphism.

Therefore $\kappa_{2}$ is injective and $H^{2}\left(M_{H}\left(r, c_{1}, \Delta\right), \mathbb{Z}\right)$ is generated by $\operatorname{im}\left(\kappa_{2}\right)$ and $\operatorname{im}(\mathfrak{a})$. By using similar computations, we see that $\kappa_{1}$ is an isomorphism. Hence Theorem 3.1 (1), (2) hold for this case.

\section{3.}

We next treat general cases. Replacing $c_{1}$ by $c_{1}+r c_{1}(H)$, we may assume that $c_{1}$ belongs to the ample cone.

Proposition 3.3. Let $(X, L)$ be a pair consisting of Abelian surface $X$ and an ample divisor $L$ of type $\left(d_{1}, d_{2}\right)$, where $d_{1}$ and $d_{2}$ are positive integers of $d_{1} \mid d_{2}$ and $\left(r, d_{1}\right)=1$. Then Theorem 3.1 (1), (2) hold for $M_{H}\left(r, c_{1}(L), \Delta\right)$, where $H$ is a general polarization.

Proof. Let $(X, L)$ be a pair consisting of Abelian surface $X$ and an ample divisor $L$ of type $\left(d_{1}, d_{2}\right)$, where $d_{1}$ and $d_{2}$ are positive integers of $d_{1} \mid d_{2}$ and $\left(r, d_{1}\right)=1$. We shall choose an ample line bundle $H$ on $X$ which is not lie on walls. Let $T$ be a connected smooth curve and $(\mathcal{X}, \mathcal{L})$ a pair of a smooth family of Abelian surface $p_{T}: \mathcal{X} \rightarrow T$ and a relatively ample line bundle $\mathcal{L}$ of type $\left(d_{1}, d_{2}\right)$. For points $t_{0}, t_{1} \in T$, we assume that $\left(\mathcal{X}_{t_{0}}, \mathcal{L}_{t_{0}}\right)=(X, L)$ and $\mathcal{X}_{t_{1}}$ is an Abelian surface of $\operatorname{NS}\left(\mathcal{X}_{t_{1}}\right) \cong \mathbb{Z}$. Let $g: \operatorname{Pic}_{\mathcal{X} / T} \rightarrow T$ be the relative Picard scheme. We denote the connected component of $\mathrm{Pic}_{\mathcal{X} / T}$ containing the section of $g$ which corresponds to the family $\mathcal{L}$ by $\operatorname{Pic}_{\mathcal{X} / T}^{\xi}$. Since $\operatorname{Pic}_{\mathcal{X} / T}^{0} \cong \operatorname{Pic}_{\mathcal{X} / T}^{\xi}, \operatorname{Pic}_{\mathcal{X} / T}^{\xi} \rightarrow T$ is a smooth morphism. Let $h: \overline{\mathcal{M}}_{\mathcal{X} / T}(r, \xi, \Delta) \rightarrow T$ be the moduli scheme parametrizing $S$-equivalence classes of $\mathcal{L}_{t}$-semi-stable sheaves $E$ on $\mathcal{X}_{t}$ with $\left(\operatorname{rk}(E), c_{1}(E), \Delta(E)\right)=\left(r, c_{1}\left(\mathcal{L}_{t}\right), \Delta\right)$ [Ma1]. Let $D$ be the closed subset of $\overline{\mathcal{M}}_{\mathcal{X} / T}(r, \xi, \Delta)$ consisting of properly $\mathcal{L}_{t}$-semi-stable sheaves on $\mathcal{X}_{t}$. Since $h$ is a proper morphism, $h(D)$ is a closed subset of $T$. Since $h(D)$ does not contain $t_{1}$ and $T$ is an irreducible curve, $h(D)$ is a finite point set. Replacing $T$ by the open subscheme $T \backslash\left(h(D) \backslash\left\{t_{0}\right\}\right)$, we may assume that $\mathcal{L}_{t}$-semi-stable sheaves are $\mathcal{L}_{t}$-stable for $t \neq t_{0}$. Let $s: \operatorname{Spl}_{\mathcal{X} / T}(r, \xi, \Delta) \rightarrow T$ be the moduli of simple sheaves $E$ on $\mathcal{X}_{t}, t \in T$ with $\left(\operatorname{rk}(E), c_{1}(E), \Delta(E)\right)=\left(r, c_{1}\left(\mathcal{L}_{t}\right), \Delta\right)$ [A-K, Thm. 7.4]. Let $U_{1}$ be the closed subset of $s^{-1}\left(T \backslash\left\{t_{0}\right\}\right)$ consisting of simple sheaves on $\mathcal{X}_{t}, t \in T \backslash\left\{t_{0}\right\}$ which are not stable with respect to $\mathcal{L}_{t}$ and 
$\overline{U_{1}}$ the closure of $U_{1}$ in $\operatorname{Spl}_{\mathcal{X} / T}(r, \xi, \Delta)$. Let $U_{2}$ be the closed subset of $s^{-1}\left(t_{0}\right)$ consisting of simple sheaves which are not semi-stable with respect to $H$. Then we can show that $\overline{U_{1}} \cap s^{-1}\left(t_{0}\right)$ is a subset of $U_{2}$ (see the second paragraph of the proof of Lemma 3.4). We set $\mathcal{M}:=\operatorname{Spl}_{\mathcal{X} / T}(r, \xi, \Delta) \backslash\left(\overline{U_{1}} \cup U_{2}\right)$. Then $\mathcal{M}$ is an open subspace of $\operatorname{Spl}_{\mathcal{X} / T}(r, \xi, \Delta)$ which is of finite type and contains all $H$-stable sheaves on $\mathcal{X}_{t_{0}}$. By using valuative criterion of separatedness and properness, we get that $s: \mathcal{M} \rightarrow T$ is a proper morphism. In fact, since $\mathcal{M} \times_{T}\left(T \backslash\left\{t_{0}\right\}\right) \rightarrow T \backslash\left\{t_{0}\right\}$ is proper, it is sufficient to check these properties near the fibre $\mathcal{X}_{t_{0}}$. The separatedness follows from base change theorem and stability with respect to $H$ (cf. [A-K, Lem. 7.8]), and the properness follows from the following lemma (Lemma 3.4) and the projectivity of $\mathcal{M}_{t_{0}}$. Since $\operatorname{Pic} c_{\mathcal{X} / T}^{\xi} \rightarrow T$ is a smooth morphism, [Mu2, Thm. 1.17] implies that $s: \mathcal{M} \rightarrow T$ is a smooth morphism. Let $\mathfrak{a}_{T}: \mathcal{M} \rightarrow \mathrm{Alb}_{\mathcal{M} / T}$ be the family of Albanese map. Let $\mathcal{F}_{T}$ be a quasi-universal family of similitude $\rho$ on $\mathcal{M} \times_{T} \mathcal{X}$ and we shall consider the homomorphism over $T$.

$$
\left\{\begin{array}{l}
\kappa_{1, t}: H^{\text {odd }}\left(\mathcal{X}_{t}, \mathbb{Z}\right) \rightarrow H^{1}\left(\mathcal{M}_{t}, \mathbb{Z}\right) \\
\kappa_{2, t}: H\left(r, c_{1}\left(\mathcal{L}_{t}\right), \Delta\right) \rightarrow H^{2}\left(\mathcal{M}_{t}, \mathbb{Z}\right)
\end{array}\right.
$$

such that $\kappa_{i, t}\left(\alpha_{i, t}\right)=\frac{1}{\rho}\left[p_{\mathcal{M}_{t *}}\left(\left(\operatorname{ch} \mathcal{F}_{t}\right) \alpha_{t}\right)\right]_{i}$, where $\alpha_{1, t} \in H^{o d d}\left(\mathcal{X}_{t}, \mathbb{Z}\right), \alpha_{2, t} \in$ $H\left(r, c_{1}\left(\mathcal{L}_{t}\right), \Delta\right)$. We assume that $\mathcal{X}_{t_{0}}$ is a product of elliptic curves. Since $p_{T}$ and $s$ are smooth, Theorem $3.1(1)$, (2) for the pair $\left(\mathcal{X}_{t_{0}}, \mathcal{L}_{t_{0}}\right)$ imply that Theorem $3.1(1)$, (2) also hold for all pairs $\left(\mathcal{X}_{t}, \mathcal{L}_{t}\right), t \in T$. By the connectedness of the moduli of $\left(d_{1}, d_{2}\right)$-polarized Abelian surfaces (cf. [L-B, $8]),(3.7)$ holds for all pairs $(X, L)$ of $\left(d_{1}, d_{2}\right)$-polarized Abelian surfaces.

LEMMA 3.4. Let $R$ be a discrete valuation ring, $K$ the quotient field of $R$, and $k$ the residue field of $R$. Let $\operatorname{Spec}(R) \rightarrow T$ be a dominant morphism such that $\operatorname{Spec}(k) \rightarrow T$ defines the point $t_{0}$. For a stable sheaf $E_{K}$ on $X_{K}$, there is a $R$-flat coherent sheaf $E$ on $X_{R}$ such that $E \otimes_{R} K=E_{K}$ and $E \otimes_{R} k$ is a $H$-stable sheaf.

If $H=\mathcal{L}_{t_{0}}$, then Langton [L] proved our claim. If $H \neq \mathcal{L}_{t_{0}}$, then we need some modifications, which will be done in the second paragraph of our proof.

Proof. Let $E^{0}$ be an $R$-flat coherent sheaf on $X_{R}$ such that $E^{0} \otimes_{R} K=$ $E_{K}$ and $E_{k}^{0}:=E^{0} \otimes_{R} k$ is torsion free. If $E_{k}^{0}$ is $H$-stable, then we put $E=E^{0}$. We assume that $E_{k}^{0}$ is not $H$-stable. Let $F_{k}^{0}\left(\subset E_{k}^{0}\right)$ be the first 
filter of the Harder-Narasimhan filtration of $E_{k}^{0}$ with respect to $H$. We set $E^{1}:=\operatorname{ker}\left(E^{0} \rightarrow E_{k}^{0} / F_{k}^{0}\right)$. Then $E^{1}$ is an $R$-flat coherent sheaf on $X_{R}$ with $E_{K}^{1}=E_{K}$. If $E_{k}^{1}$ is not $H$-stable, then we shall consider the first filter $F_{k}^{1}$ of the Harder-Narasimhan filtration of $E_{k}^{1}$ and set $E^{2}:=\operatorname{ker}\left(E^{1} \rightarrow E_{k}^{1} / F_{k}^{1}\right)$. Continuing this procedure successively, we obtain a decreasing sequence of $R$-flat coherent sheaves on $X_{R}: E^{0} \supset E^{1} \supset E^{2} \supset \cdots$. We assume that this sequence is infinite. Then in the same way as in [L, Lem. 2], we see that there is an integer $i$ such that $E^{i} \otimes_{R} \widehat{R}$ has a subsheaf $F$ of rank $r^{\prime}$ with $F \otimes_{R} k=F_{k}^{i}$, where $\widehat{R}$ is the completion of $R$.

We set $\widehat{K}:=K \otimes_{R} \widehat{R}$ and $D:=\operatorname{det}\left(E^{i} \otimes_{R} \widehat{R}\right)^{\otimes r^{\prime}} \otimes \operatorname{det}(F)^{\otimes(-r)}$. Let $P(x)$ be the Hilbert polynomial of $D$ with respect to $\mathcal{L}_{\widehat{R}}$. Let $V$ be a locally free sheaf on $\mathcal{X}$ such that there is a surjective homomorphism $V \otimes_{\mathcal{O}_{T}} \widehat{R} \rightarrow D$, and we shall consider the quot scheme $\mathcal{Q}:=\operatorname{Quot}_{V / \mathcal{X} / T}^{P(x)}$. Then $D$ defines a morphism $\tau: \operatorname{Spec}(\widehat{R}) \rightarrow \mathcal{Q}$ such that $D=\left(\tau \times_{T} 1_{\mathcal{X}}\right)^{*} \mathcal{D}$, where $\mathcal{D}$ is the universal quotient. Let $\mathcal{Q}_{0}$ be the connected component of $\mathcal{Q}$ which contains the image of $\operatorname{Spec}(\widehat{R})$. Since $\operatorname{Spec}(\widehat{R}) \rightarrow T$ is dominant, $\mathfrak{q}: \mathcal{Q}_{0} \rightarrow T$ is dominant, and hence surjective. Since $E_{\widehat{K}}^{i} \cong E_{K} \otimes_{K} \widehat{K}$ is a stable sheaf on $X_{\widehat{K}},\left(\mathcal{D}_{q_{1}}, \mathcal{L}_{q_{1}}\right)=\left(\mathcal{D}_{\widehat{K}}, \mathcal{L}_{\widehat{K}}\right)>0$, where $q_{1}$ is a point of $\mathfrak{q}^{-1}\left(t_{1}\right)$. Since $\operatorname{NS}\left(\mathcal{X}_{t_{1}}\right) \cong \mathbb{Z}$, we get that $c_{1}\left(\mathcal{D}_{q_{1}}\right)=l c_{1}\left(\mathcal{L}_{q_{1}}\right), l>0$. Hence we obtain that $\left(\mathcal{D}_{\tau\left(t_{0}\right)}^{2}\right)>0$ and $\left(\mathcal{D}_{\tau\left(t_{0}\right)}, \mathcal{L}_{\tau\left(t_{0}\right)}\right)>0$. By the Riemann-Roch theorem and the Serre duality, we see that $\mathcal{D}_{\tau\left(t_{0}\right)}$ is an effective divisor. Therefore $\left(\mathcal{D}_{\tau\left(t_{0}\right)}, H\right)>0$, which is a contradiction. Hence there is an integer $n$ such that $E^{n} \otimes_{R} k$ is $H$-stable.

Proof of Theorem $3.1(3)$. Let $\kappa_{2}^{\prime}: H\left(r, c_{1}, \Delta\right) \otimes \mathbb{C} \rightarrow H^{2}\left(M\left(r, c_{1}, \Delta\right), \mathbb{C}\right)$ be the homomorphism induced by $\kappa_{2}$. We note that $H^{2,0}(X)$ and $H^{0,2}(X)$ are subsets of $H\left(r, c_{1}, \Delta\right) \otimes \mathbb{C}$. Since $\operatorname{ch}_{i}(\mathcal{F})$ is of type $(i, i)$, we see that

$$
\left\{\begin{array}{l}
\kappa_{2}^{\prime}\left(H^{2,0}(X)\right) \subset H^{2,0}\left(M_{H}\left(r, c_{1}, \Delta\right)\right) \\
\kappa_{2}^{\prime}\left(\bigoplus_{p=0}^{2} H^{p, p}(X)\right) \subset H^{1,1}\left(M_{H}\left(r, c_{1}, \Delta\right)\right) \\
\kappa_{2}^{\prime}\left(H^{0,2}(X)\right) \subset H^{0,2}\left(M_{H}\left(r, c_{1}, \Delta\right)\right) .
\end{array}\right.
$$

Since $H\left(r, c_{1}, \Delta\right) \otimes \mathbb{C}=H^{2,0}(X) \oplus\left(\bigoplus_{p=0}^{2} H^{p, p}(X)\right) \cap H\left(r, c_{1}, \Delta\right) \otimes \mathbb{C} \oplus$ $H^{0,2}(X)$ and $\mathfrak{a}^{*}$ preserves the type, we obtain that

$$
\begin{gathered}
H^{1,1}\left(M_{H}\left(r, c_{1}, \Delta\right)\right)=\kappa_{2}^{\prime}\left(\left(\bigoplus_{p=0}^{2} H^{p, p}(X)\right) \cap H\left(r, c_{1}, \Delta\right) \otimes \mathbb{C}\right) \\
\oplus \mathfrak{a}^{*}\left(H^{1,1}\left(\operatorname{Alb}\left(M_{H}\left(r, c_{1}, \Delta\right)\right)\right)\right) .
\end{gathered}
$$


Hence we get Theorem 3.1 (3).

Combining [Y4, Thm. 2.1] with the proof of Proposition 3.3, we get the following theorem.

TheOrem 3.5. Let $X$ be an Abelian surface defined over $\mathbb{C}$ and $c_{1} \in$ $\mathrm{NS}(X)$ a primitive element. Then

$$
P\left(M_{H}\left(2, c_{1}, \Delta\right), z\right)=P\left(M_{H}(1,0,2 \Delta), z\right)
$$

for a general polarization $H$, where $P(\cdot, z)$ is the Poincaré polynomial.

\section{4.}

We shall next consider the Albanese variety of $M_{H}\left(r, c_{1}, \Delta\right)$, if $\operatorname{dim}$ $M_{H}\left(r, c_{1}, \Delta\right) \geq 4$. Let $\mathcal{P}$ be the Poincaré line bundle on $\widehat{X} \times X$, where $\widehat{X}$ is the dual of $X$. For an element $E_{0} \in M_{H}\left(r, c_{1}, \Delta\right)$, let $\alpha_{E_{0}}: M_{H}\left(r, c_{1}, \Delta\right) \rightarrow$ $X$ be the morphism sending $E \in M_{H}\left(r, c_{1}, \Delta\right)$ to $\operatorname{det} p_{\widehat{X} !}\left(\left(E-E_{0}\right) \otimes(\mathcal{P}-\right.$ $\left.\left.\mathcal{O}_{\widehat{X} \times X}\right)\right) \in \operatorname{Pic}^{0}(\widehat{X})=X$, and $\operatorname{det}_{E_{0}}: M_{H}\left(r, c_{1}, \Delta\right) \rightarrow \widehat{X}$ the morphism sending $E$ to $\operatorname{det} E \otimes \operatorname{det} E_{0}^{\vee} \in \widehat{X}$ (cf. [Y3, Sect. 5]). We shall show that $\mathfrak{a}_{E_{0}}:=\operatorname{det}_{E_{0}} \times \alpha_{E_{0}}$ is the Albanese map of $M_{H}\left(r, c_{1}, \Delta\right)$. Let $B$ be an effective divisor on $X$. Then we see that

$$
\begin{aligned}
& \operatorname{det} p_{\widehat{X} !}\left(\left(E-E_{0}\right) \otimes \mathcal{O}_{B} \otimes\left(\mathcal{P}-\mathcal{O}_{\widehat{X} \times X}\right)\right) \\
& \quad=\operatorname{det} p_{\widehat{X} !}\left(\left(\left.\operatorname{det} E\right|_{B}-\left.\operatorname{det} E_{0}\right|_{B}\right) \otimes\left(\mathcal{P}-\mathcal{O}_{\widehat{X} \times X}\right)\right) \\
& \quad=\zeta\left(\operatorname{det}_{E_{0}}(E)\right),
\end{aligned}
$$

where $\zeta: \widehat{X} \rightarrow X$ is the morphism sending $L \in \widehat{X}$ to $\bigotimes_{i} \mathcal{P}_{\widehat{X} \times\left\{x_{i}\right\}} \in$ $\operatorname{Pic}^{0}(\widehat{X})=X, L \cdot B=\sum_{i} x_{i}$. Therefore if $\mathfrak{a}_{E_{0}}$ is the Albanese map for $M_{H}\left(r, c_{1}, \Delta\right)$, then $\mathfrak{a}_{E_{0}(B)}$ is the Albanese map for $M_{H}\left(r, c_{1}+r c_{1}\left(\mathcal{O}_{X}(B)\right), \Delta\right)$. Hence we may assume that $c_{1}$ belongs to the ample cone. In the notation of Proposition 3.3, we assume that there is a section $\sigma: T \rightarrow \mathcal{M}$ of $s$. Then we can also construct a morphism $\mathfrak{a}_{\sigma}: \mathcal{M} \rightarrow \operatorname{Pic}_{\mathcal{X} / T}^{0} \times_{T} \mathcal{X}$. In fact, it is sufficient to construct the morphism on small neighbourhoods $U$ (in the sense of classical topology) of each point. By using a universal family on $U \times_{T} \mathcal{X}$, we get the morphism. Since $s: \mathcal{M} \rightarrow T$ and $\operatorname{Pic}_{\mathcal{X} / T}^{0} \times_{T} \mathcal{X} \rightarrow T$ are smooth over $T$, it is sufficient to prove that

$$
\mathfrak{a}_{E_{0}}^{*}: H^{1}(\widehat{X} \times X, \mathbb{Z}) \longrightarrow H^{1}\left(M_{H}\left(r, c_{1}, \Delta\right), \mathbb{Z}\right)
$$


is an isomorphism, if $X$ is a product of elliptic curves. In order to prove this assertion, we shall show that

$$
\mathfrak{a}_{E_{0}}^{*}: \operatorname{Pic}^{0}(\widehat{X} \times X) \longrightarrow \operatorname{Pic}^{0}\left(M\left(r, c_{1}, \Delta\right)\right)
$$

is an isomorphism. Let $\mathcal{E}$ be a universal family on $M\left(r, c_{1}, \Delta\right)$. For simplicity, we set $M:=M\left(r, c_{1}, \Delta\right)$. Let $\widehat{X} \times X \rightarrow \operatorname{Pic}^{0}(X \times \widehat{X})$ be the isomorphism sending $(\hat{x}, x) \in \widehat{X} \times X$ to $\left.\left.\mathcal{P}\right|_{\{\hat{x}\} \times X} \otimes \mathcal{P}\right|_{\widehat{X} \times\{x\}}$. We set $\mathcal{R}:=\operatorname{det} p_{\widehat{X} \times M !}((\mathcal{E}-$ $\left.\left.E_{0} \otimes \mathcal{O}_{M}\right) \otimes\left(\mathcal{P}-\mathcal{O}_{\widehat{X} \times X}\right)\right)$. By the construction of $\alpha_{E_{0}}$, we get that $\mathcal{R} \cong$ $\left(1_{\widehat{X}} \times \alpha_{E_{0}}\right)^{*} \mathcal{P} \otimes L$, where $L$ is the pull-back of a line bundle on $M$. Since $\left.\mathcal{R}\right|_{\{0\} \times M} \cong \mathcal{O}_{M}$, we get that $L \cong \mathcal{O}_{\widehat{X} \times M}$. Hence we see that

$$
\begin{aligned}
\alpha_{E_{0}}^{*}\left(\left.\mathcal{P}\right|_{\{\hat{x}\} \times X}\right) & =\operatorname{det} p_{M !}\left(\left(\mathcal{E}-E_{0} \otimes \mathcal{O}_{M}\right) \otimes\left(\left.\mathcal{P}\right|_{\{\hat{x}\} \times X}-\mathcal{O}_{X}\right)\right) \\
& =\operatorname{det} p_{M !}\left(\mathcal{E} \otimes\left(\left.\mathcal{P}\right|_{\{\hat{x}\} \times X}-\mathcal{O}_{X}\right)\right)
\end{aligned}
$$

In the same way, we see that

$$
\begin{aligned}
\operatorname{det}_{E_{0}}^{*}\left(\left.\mathcal{P}\right|_{\widehat{X} \times\{x\}}\right) & =\left.\left(\left.\operatorname{det} \mathcal{E} \otimes \operatorname{det} E_{0}^{\vee} \otimes \operatorname{det} \mathcal{E}^{\vee}\right|_{M \times\{0\}}\right)\right|_{M \times\{x\}} \\
& =\operatorname{det} p_{M !}\left(\mathcal{E} \otimes\left(k_{x}-k_{0}\right)\right),
\end{aligned}
$$

where $0 \in X$ is the zero of the group low. In order to prove (3.27), we shall consider the pull-backs of $\alpha_{E_{0}}^{*}\left(\left.\mathcal{P}\right|_{\{\hat{x}\} \times X}\right)$ and $\operatorname{det}_{E_{0}}^{*}\left(\left.\mathcal{P}\right|_{\widehat{X} \times\{x\}}\right)$ to $X^{0} \times Y_{0}$.

We denote the zero of $C_{1}$ and $C_{2}$ by $0_{1}$ and $0_{2}$ respectively. For a point $q_{k}$ of $C_{k}, k=1,2$, we set $l_{k}:=q_{k}-0_{k}$. We also denote the pull-back of $l_{k}$ to $X=C_{1} \times C_{2}$ by $l_{k}$. In the same way as in $\mathbf{3 . 2}$, we denote $\varpi_{i}^{!}(G)$, $i=0,1, \ldots, n, a$ by $G^{i}, G \in K(X)$. We also denote $\mathcal{O}_{X}(D)^{i}$ by $\mathcal{O}_{X^{0} \times Y_{0}}\left(D^{i}\right)$. By simple calculations, we see that

$$
\left\{\begin{array}{l}
\operatorname{det} p_{X^{0} \times Y_{0} !}\left(\mathcal{E} \otimes\left(\mathcal{O}_{X}\left(l_{1}\right)-\mathcal{O}_{X}\right)^{a}\right)=\mathcal{O}_{X^{0} \times Y_{0}}\left(d l_{1}^{0}-d_{2} \sum_{i=1}^{n} l_{1}^{i}\right) \\
\operatorname{det} p_{X^{0} \times Y_{0} !}\left(\mathcal{E} \otimes\left(\mathcal{O}_{X}\left(l_{2}\right)-\mathcal{O}_{X}\right)^{a}\right)=\mathcal{O}_{X^{0} \times Y_{0}}\left(\sum_{i=1}^{n} l_{2}^{i}\right) \\
\operatorname{det} p_{X^{0} \times Y_{0} !}\left(\mathcal{E} \otimes\left(k_{\left(q_{1}, 0_{2}\right)}-k_{\left(0_{1}, 0_{2}\right)}\right)^{a}\right)=\mathcal{O}_{X^{0} \times Y_{0}}\left(r l_{1}^{0}-r_{2} \sum_{i=1}^{n} l_{1}^{i}\right) \\
\operatorname{det} p_{X^{0} \times Y_{0} !}\left(\mathcal{E} \otimes\left(k_{\left(0_{1}, q_{2}\right)}-k_{\left(0_{1}, 0_{2}\right)}\right)^{a}\right)=\mathcal{O}_{X^{0} \times Y_{0}}\left(l_{2}^{0}\right) .
\end{array}\right.
$$

Since $d_{2} r-d r_{2}=1$ and $\operatorname{Pic}^{0}\left(X^{0} \times \operatorname{Hilb}_{X}^{n}\right) \cong \operatorname{Pic}^{0}\left(X^{0} \times Y_{0}\right)^{\mathfrak{S}_{n}},(3.28),(3.29)$ and (3.30) implies that (3.27) holds. 
We set

$$
K\left(r, c_{1}, \Delta\right):=\left\{\alpha \in K(X) \mid \chi\left(\alpha \otimes E_{0}\right)=0, E_{0} \in M_{H}\left(r, c_{1}, \Delta\right)\right\} .
$$

Let $\left\{U_{i}\right\}$ be an open covering of $M_{H}\left(r, c_{1}, \Delta\right)$ such that there are universal family $\mathcal{F}_{i}$ on each $U_{i} \times X$ and $\left.\left.\mathcal{F}_{i}\right|_{\left(U_{i} \cap U_{j}\right) \times X} \cong \mathcal{F}_{j}\right|_{\left(U_{i} \cap U_{j}\right) \times X}$. Since the action of $\mathcal{O}_{U_{i}}^{\times}$to det $p_{U_{i} !}\left(\mathcal{F}_{i} \otimes \alpha\right)$ is trivial, we get a line bundle $\tilde{\kappa}(\alpha)$ on $M_{H}\left(r, c_{1}, \Delta\right)$. Thus we obtain a homomorphism

$$
\tilde{\kappa}: K\left(r, c_{1}, \Delta\right) \longrightarrow \operatorname{Pic}\left(M_{H}\left(r, c_{1}, \Delta\right)\right) .
$$

We note that there is a commutative diagram:

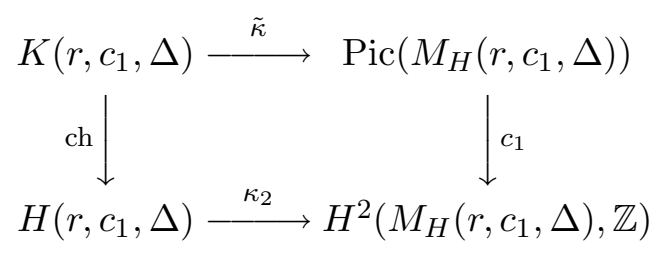

Let $K^{2}$ be the subgroup of $K\left(r, c_{1}, \Delta\right)$ generated by $k_{P}-k_{0}, P \in X$ and $N$ the kernel of the Albanese map $K^{2} \rightarrow X$. Since ker (ch) is generated by $\mathcal{O}_{X}(D)-\mathcal{O}_{X}, \mathcal{O}_{X}(D) \in \operatorname{Pic}^{0}(X)$ and $k_{P}-k_{0}, P \in X,(3.28)$ and (3.29) implies that $\tilde{\kappa}$ induces an isomorphism $\operatorname{ker}(\operatorname{ch}) / N \rightarrow \operatorname{Pic}^{0}\left(M_{H}\left(r, c_{1}, \Delta\right)\right)$. By using Theorem 3.1 (3), we get the following theorem, which is similar to $[\mathrm{Y} 2$, Thm. 0.1].

TheOREM 3.6. Under the same assumption as in Theorem 3.1, the following holds.

(1) $\mathfrak{a}_{E_{0}}: M_{H}\left(r, c_{1}, \Delta\right) \rightarrow \widehat{X} \times X$ is an Albanese map.

(2) $\tilde{\kappa}: K\left(r, c_{1}, \Delta\right) / N \rightarrow \operatorname{Pic}\left(M_{H}\left(r, c_{1}, \Delta\right)\right)$ is injective.

(3) $\operatorname{Pic}\left(M_{H}\left(r, c_{1}, \Delta\right)\right) / \mathfrak{a}_{E_{0}}^{*}(\operatorname{Pic}(\widehat{X} \times X))$ is generated by $\tilde{\kappa}\left(K\left(r, c_{1}, \Delta\right)\right)$.

(4) $\mathfrak{a}_{E_{0}}^{*}(\operatorname{Pic}(\widehat{X} \times X)) \cap \tilde{\kappa}\left(K\left(r, c_{1}, \Delta\right)\right) \cong X \times \widehat{X}$.

\section{$\S 4$. Appendix}

In this appendix, we shall show the following. We shall also show that $M_{H}\left(r, c_{1}, \Delta\right) \cong \widehat{X} \times X$, if $\operatorname{dim} M_{H}\left(r, c_{1}, \Delta\right)=4$.

Proposition 4.1. Let $L$ be an ample line bundle on an Abelian surface $X$. We assume that $\chi(L)=\left(c_{1}(L)^{2}\right) / 2$ and $r$ are relatively prime. Then $M_{H}\left(r, c_{1}(L), \Delta\right) \cong M_{H}(r, L, \Delta) \times \widehat{X}$, where $M_{H}(r, L, \Delta)$ is the moduli space of sheaves of determinant $L$. In particular, $P\left(M_{H}(2, L, \Delta), z\right)=$ $P\left(\operatorname{Hilb}_{X}^{2 \Delta}, z\right)$ for a general polarization $H$. 
Proof. For a stable sheaf $E \in M_{H}\left(r, c_{1}(L), \Delta\right), \lambda(E)$ denotes the point of $\widehat{X}$ which correspond to the line bundle $\operatorname{det}(E) \otimes L^{-1}$. Let $\phi_{L}: X \rightarrow \widehat{X}$ be the morphism sending $x \in X$ to $T_{x}^{*} L \otimes L^{-1}$, and $\varphi: \widehat{X} \rightarrow X$ the morphism such that $\phi_{L} \circ \varphi=n_{\widehat{X}}^{2}$, where $T_{x}: X \rightarrow X$ is the translation defined by $x$ and $n^{2}=\chi(L)^{2}=\operatorname{deg} \phi_{L}$. Since $\left(r, n^{2}\right)=1$, there are integers $k$ and $k^{\prime}$ such that $r k+n^{2} k^{\prime}=1$. We denote the Poincaré line bundle on $X \times \widehat{X}$ by $\mathcal{P}$. Let $A: M_{H}\left(r, c_{1}(L), \Delta\right) \rightarrow M_{H}(r, L, \Delta) \times \widehat{X}$ be the morphism sending $F \in$ $M_{H}\left(r, c_{1}(L), \Delta\right)$ to $\left(T_{-k^{\prime} \varphi \circ \lambda(F)}^{*}\left(F \otimes \mathcal{P}_{-k \lambda(F)}\right), \lambda(F)\right)$ and $B: M_{H}(r, L, \Delta) \times$ $\widehat{X} \rightarrow M_{H}\left(r, c_{1}(L), \Delta\right)$ the morphism sending $(E, x) \in M_{H}(r, L, \Delta) \times \widehat{X}$ to $T_{k^{\prime} \varphi(X)}^{*} E \otimes \mathcal{P}_{k x}$. For an element $(E, x)$ of $M_{H}(r, L, \Delta) \times \widehat{X}, \operatorname{det}\left(T_{k^{\prime} \varphi(x)}^{*} E \otimes\right.$ $\left.\mathcal{P}_{k x}\right) \cong T_{k^{\prime} \varphi(x)}^{*} L \otimes \mathcal{P}_{r k x} \cong L \otimes \mathcal{P}_{k^{\prime} \phi_{L^{\circ}} \varphi(x)} \otimes \mathcal{P}_{r k x} \cong L \otimes \mathcal{P}_{\left(n^{2} k^{\prime}+r k\right) x}=L \otimes \mathcal{P}_{x}$. Hence $\lambda \circ B((E, x))=x$. Then it is easy to see that $A \circ B$ and $B \circ A$ are identity morphisms. Hence $A: M_{H}\left(r, c_{1}(L), \Delta\right) \rightarrow M_{H}(r, L, \Delta) \times \widehat{X}$ is an isomorphism.

Let $\mathbb{D}(X)$ and $\mathbb{D}(\widehat{X})$ be the derived categories of $X$ and $\widehat{X}$ respectively. Let $\mathcal{S}: \mathbb{D}(X) \rightarrow \mathbb{D}(\widehat{X})$ be the Fourier-Mukai transform [Mu4]. Then the morphism $\alpha:=\alpha_{E_{0}}$ defined in 3.4 satisfies that $\alpha(E)=\operatorname{det} \mathcal{S}(E) \otimes$ $\left(\operatorname{det} \mathcal{S}\left(E_{0}\right)\right)^{-1}$. Thus $\alpha_{E_{0}}$ is also defined by Fourier-Mukai transform. By using [Mu4], we shall treat the case $2 r \Delta=2$ (at least, Mukai treated the case where $X$ is a principally polarized Abelian surface).

Proposition 4.2. Let $L$ be an ample divisor. If $2 r \Delta=2$, then for a general polarization $H$, the Albanese map $\alpha: M_{H}(r, L, \Delta) \rightarrow X$ is an isomorphism.

Proof. Since $r c_{2}-(r-1)\left(L^{2}\right) / 2=1$ and $\chi(L)=\left(L^{2}\right) / 2, r$ and $\chi(L)$ are relatively prime. We shall choose an element $E$ of $M_{H}(r, L, \Delta)$ and let $\xi: X \times \widehat{X} \rightarrow M\left(r, c_{1}(L), \Delta\right)$ be the morphism sending $(x, y) \in X \times \widehat{X}$ to $T_{x}^{*} E \otimes \mathcal{P}_{y}$. Then $\lambda \circ \xi(x, y)=\phi_{L}(x)+r y$. Let $f: X \rightarrow X \times \widehat{X}$ be the morphism such that $f(x)=\left(r x,-\phi_{L}(x)\right)$. Since $\# \operatorname{ker} \phi_{L}=\chi(L)^{2}$ and $r$ are relatively prime, $f$ is injective. Let $g: \widehat{X} \rightarrow X \times \widehat{X}$ be the morphism such that $g(y)=$ $\left(k^{\prime} \varphi(y), k y\right)$. Then $f \times g: X \times \widehat{X} \rightarrow X \times \widehat{X}$ is an isomorphism. In fact, if $\left(r x+k^{\prime} \varphi(y),-\phi_{L}(x)+k y\right)=(0,0)$, then $\phi_{L}\left(r x+k^{\prime} \varphi(y)\right)=r \phi_{L}(x)+n^{2} k^{\prime} y=$ 0 . Hence $y=\left(n^{2} k^{\prime}+r k\right) y=0$. Since $f$ is injective, $x=0$, which implies that $f \times g$ is injective. Therefore $f \times g$ is an isomorphism. Then we get a morphism $\xi \circ f: X \rightarrow M(r, L, \Delta)$. Replacing $E$ by $E \otimes L^{\otimes m}$, we may assume that there is an exact sequence $0 \rightarrow \mathcal{O}_{X}^{\oplus(r-1)} \rightarrow E \rightarrow I_{Z} \otimes L \rightarrow 0$, where $I_{Z}$ 
is the ideal sheaf of a codimension 2 subscheme $Z$ of $X$. By our assumption on Chern classes, $1 / r=\Delta(E)=\operatorname{deg} Z-(r-1) / r \chi(L)$. For simplicity, we denote $\operatorname{det} \mathcal{S}(\cdot)$ by $\delta(\cdot)$. Then we see that $\delta\left(T_{x}^{*} E \otimes \mathcal{P}_{y}\right)=\delta\left(I_{T_{-x}(Z)} \otimes T_{x}^{*} L \otimes\right.$ $\left.\mathcal{P}_{y}\right)=\delta\left(I_{Z-(\operatorname{deg} Z) x} \otimes L \otimes \mathcal{P}_{\phi_{L}(x)+y}\right)=\delta\left(L \otimes \mathcal{P}_{\phi_{L}(x)+y}\right) \otimes \mathcal{P}_{-Z+(\operatorname{deg} Z) x}=$ $\operatorname{det} T_{\phi_{L}(x)+y}^{*}(\mathcal{S}(L)) \otimes \mathcal{P}_{-Z+(\operatorname{deg} Z) x}=\delta(L) \otimes \mathcal{P}_{\phi_{\delta(L)}}\left(\phi_{L}(x)+y\right)+(\operatorname{deg} Z) x-Z$. Hence $\alpha \circ \xi \circ f(x)=\alpha \circ \xi \circ f(0)+(r-1) \phi_{\delta(L)} \circ \phi_{L}(x)+r(\operatorname{deg} Z) x$. By the proof of [Mu4, Prop. 1.23], $\phi_{\delta(L)}\left(\phi_{L}(x)\right)=-\chi(L) x$. Since $r \operatorname{deg} Z=1+(r-1) \chi(L)$, we get that $\alpha \circ \xi \circ f(x)=\alpha \circ \xi \circ f(0)+x$. Thus $\alpha \circ \xi \circ f(x)$ is an isomorphism. Therefore we get that $\alpha: M_{H}(r, L, \Delta) \rightarrow X$ is an isomorphism.

Corollary 4.3. $M_{H}\left(r, c_{1}, \Delta\right) \cong \widehat{X} \times X$, if $\operatorname{dim} M_{H}\left(r, c_{1}, \Delta\right)=4$.

\section{REFERENCES}

[A-K] A. Altman and S. Kleiman, Compactifying the Picard scheme, Adv. in Math., 35 (1980), 50-112.

[A] M. F. Atiyah, Vector bundles over an elliptic curve, Proc. Lond. Math. Soc. (3) VII, 2 (1957), 414-452.

[A-B] M. F. Atiyah and R. Bott, The Yang-Mills equations over Riemann surfaces, Philos. Trans. Roy. Soc. London Ser. A, 308 (1982), 523-615.

[D] J.-M. Drezet, Points non factoriels des variétés de modules de faisceaux semi-stable sur une surface rationelle, J. reine angew. Math., 413 (1991), 99-126.

[D-N] J.-M. Drezet and M. S. Narasimhan, Groupe de Picard des variétés de modules de fibrés semi-stables sur les courbes algébriques, Invent. Math., 97 (1989), 53-94.

[F] R. Friedman, Vector bundles and SO(3)-invariants for elliptic surfaces III, Preprint (1993).

[G-H] L. Göttsche and D. Huybrechts, Hodge numbers of moduli spaces of stable bundles on K3 surfaces, Preprint (1994).

[K] D. Knutson, Algebraic Spaces, Lecture Notes in Math. 203, Springer-Verlag.

[L] S. G. Langton, Valuative criteria for families of vector bundles on an algebraic varieties, Ann. of Math., 101 (1975), 88-110.

[L-B] H. Lange and Ch. Birkenhake, Complex Abelian Varieties, Springer-Verlag.

[Li1] J. Li, The first two Betti numbers of the moduli spaces of vector bundles on surfaces, Preprint (1995).

[Li2] J. Li, Picard groups of the moduli spaces of vector bundles over algebraic surfaces, moduli of vector bundles, Lect. Notes in Pure and Applied Math. 179, Marcel Deckker, pp. 129-146.

[Ma1] M. Maruyama, Moduli of stable sheaves II, J. Math. Kyoto Univ., 18 (1978), 557-614.

[Ma2] M. Maruyama, Moduli of algebraic vector bundles, in preparation.

[Mu1] S. Mukai, Semi-homogeneous vector bundles on an abelian variety, J. Math. Kyoto Univ., 18 (1978), 239-272. 
[Mu2] S. Mukai, Symplectic structure of the moduli space of sheaves on an abelian or K3 surface, Invent. Math., 77 (1984), 101-116.

[Mu3] S. Mukai, On the moduli space of bundles on K3 surfaces I, Vector bundles on Algebraic Varieties (1987), 341-413.

[Mu4] S. Mukai, Fourier functor and its application to the moduli of bundles on an Abelian variety, Adv. Studies in Pure Math., 10 (1987), 515-550.

[Mu5] S. Mukai, Moduli of vector bundles on K3 surfaces, and symplectic manifolds, Sugaku Expositions, 1 (1988), 139-174.

[O] K. O'Grady, The weight-two Hodge structure of moduli spaces of sheaves on a K3 surface, Preprint (1995).

[S] C. Simpson, Moduli of representations of the fundamental group of a smooth projective variety I, Publ. Math. I.H.E.S., 79 (1994), 47-129.

[Y1] K. Yoshioka, The Betti numbers of the moduli space of stable sheaves of rank 2 on $\mathbb{P}^{2}$, J. reine angew. Math., 453 (1994), 193-220.

[Y2] K. Yoshioka, The Picard group of the moduli space of stable sheaves on a ruled surface, J. Math. Kyoto Univ., 36 (1996), 279-309.

[Y3] K. Yoshioka, Chamber structure of polarizations and the moduli of stable sheaves on a ruled surface, Internat. J. Math., 7 (1996), 411-431.

[Y4] K. Yoshioka, Numbers of $\mathbb{F}_{q}$-rational points of moduli of stable sheaves on elliptic surfaces, moduli of vector bundles, Lect. Notes in Pure and Applied Math. 179, Marcel Deckker, pp. 297-305.

Department of Mathematics

Kobe University

Kobe, 65\%-8501

Japan

yoshioka@math.s.kobe-u.ac.jp 